\title{
A VLA Study of High-redshift GRBs. I. Multiwavelength Observations and Modeling of GRB 140311A
}

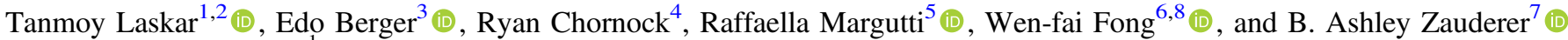 \\ ${ }^{1}$ National Radio Astronomy Observatory, 520 Edgemont Road, Charlottesville, VA 22903, USA \\ ${ }^{2}$ Department of Astronomy, University of California, 501 Campbell Hall, Berkeley, CA 94720-3411, USA \\ ${ }^{3}$ Department of Astronomy, Harvard University, 60 Garden Street, Cambridge, MA 02138, USA \\ ${ }^{4}$ Astrophysical Institute, Department of Physics and Astronomy, 251B Clippinger Lab, Ohio University, Athens, OH 45701, USA \\ ${ }^{5}$ Center for Interdisciplinary Exploration and Research in Astrophysics (CIERA) and Department of Physics and Astrophysics, \\ Northwestern University, Evanston, IL 60208, USA \\ ${ }^{6}$ Steward Observatory, University of Arizona, 933 N. Cherry Avenue, Tucson, AZ 85721, USA \\ ${ }^{7}$ Center for Cosmology and Particle Physics, New York University, 4 Washington Place, New York, NY 10003, USA \\ Received 2017 July 14; revised 2018 March 12; accepted 2018 March 13; published 2018 May 4
}

\begin{abstract}
We present the first results from a recently concluded study of GRBs at $z \gtrsim 5$ with the Karl G. Jansky Very Large Array (VLA). Spanning 1 to $85.5 \mathrm{GHz}$ and 7 epochs from 1.5 to 82.3 days, our observations of GRB $140311 \mathrm{~A}$ are the most detailed joint radio and millimeter observations of a GRB afterglow at $z \gtrsim 5$ to date. In conjunction with optical/near-IR and X-ray data, the observations can be understood in the framework of radiation from a single blast wave shock with energy $E_{\mathrm{K} \text {,iso }} \approx 8.5 \times 10^{53} \mathrm{erg}$ expanding into a constant density environment with density, $n_{0} \approx 8 \mathrm{~cm}^{-3}$. The $\mathrm{X}$-ray and radio observations require a jet break at $t_{\mathrm{jet}} \approx 0.6$ days, yielding an opening angle of $\theta_{\text {jet }} \approx 4^{\circ}$ and a beaming-corrected blast wave kinetic energy of $E_{\mathrm{K}} \approx 2.2 \times 10^{50} \mathrm{erg}$. The results from our radio follow-up and multiwavelength modeling lend credence to the hypothesis that detected high-redshift GRBs may be more tightly beamed than events at lower redshift. We do not find compelling evidence for reverse shock emission, which may be related to fast cooling driven by the moderately high circumburst density.
\end{abstract}

Key words: gamma-ray burst: general - gamma-ray burst: individual (140311A)

Supporting material: machine-readable tables

\section{Introduction}

Long-duration $\gamma$-ray bursts (GRBs) have been firmly established to originate from the catastrophic death of massive stars (e.g., Woosley \& Bloom 2006). The large luminosities of GRB afterglows make these energetic events premier probes of the high-redshift universe, ranging from the parsec-scale environments of the progenitors to the properties of the intergalactic medium (Totani et al. 2006; Inoue et al. 2007; Tanvir et al. 2009; Wang et al. 2012; Chornock et al. 2013, 2014). Additionally, high-redshift GRBs have been speculated to possibly differ from lower redshift events in their energy scales, durations, and circumburst media (Fryer et al. 2001; Bromm et al. 2003; Heger et al. 2003; Suwa \& Ioka 2011; Toma et al. 2011). Due to time dilation, high-redshift GRBs also afford an opportunity to capture rapidly evolving reverse shock emission, and thereby a means of probing the Lorentz factor and composition of the relativistic ejecta powering the afterglow (Piran 2005; Mészáros 2006; Laskar et al. 2013, 2014, 2016; Perley et al. 2014; Alexander et al. 2017).

Detailed multiwavelength observations of GRB afterglows spanning the X-rays to the radio bands yield a measurement of the explosion properties and circumburst environments (e.g., Sari et al. 1998). Whereas the rapid response of Swift has yielded prompt X-ray afterglow localization and rich X-ray light curves, and ground-based facilities have improved the detection and characterization of optical light curves (Nousek et al. 2006; Liang et al. 2007, 2008; Evans et al. 2009; Margutti et al. 2013; Zaninoni et al. 2013), detailed observations of afterglows in the radio and millimeter have resulted in a low

\footnotetext{
${ }^{8}$ Einstein Fellow.
}

detection rate of about $30 \%$, with sensitivity being the primary challenge (Chandra \& Frail 2012; de Ugarte Postigo et al. 2012).

Due to these limitations, the multiwavelength properties of GRBs at $z \gtrsim 5$ remain poorly characterized (Tagliaferri et al. 2005; Haislip et al. 2006; Kawai et al. 2006; Greiner et al. 2009; Salvaterra et al. 2009; Tanvir et al. 2009; Cucchiara et al. 2011). We carried out a comprehensive analysis of three GRBs at $z \gtrsim 6$ with radio detections and demonstrated that these events exhibit explosion energies typical of the lower redshift population but tend to exhibit narrower jet opening angles (Laskar et al. 2014, henceforth LBT14). However, the radio observations for two out of the three events only yielded an upper bound on the synchrotron self-absorption frequency, resulting in an order of magnitude or greater uncertainty in the circumburst density and degeneracies between the various physical parameters.

With the upgrade of the Karl G. Jansky Very Large Array (VLA) providing an order of magnitude improvement in sensitivity and continuous frequency coverage from 1 to $40 \mathrm{GHz}$, detailed observations of GRB afterglows in the $\mathrm{cm}$ band are now feasible. Taking advantage of this opportunity, we targeted all events with secure spectroscopic redshifts of $z \gtrsim 5$ at multiple VLA frequencies, supported by millimeterband data from the Combined Array for Millimeter Astronomy (CARMA). Our sample consists of four events: GRBs 130606A, 140304A, 140311A, and 140515A. In this series of papers, we present the results of our observations and characterize the multiwavelength afterglows of high-redshift GRBs, focusing, in particular, on whether the inferred explosion properties and circumburst environments are 
suggestive of evolution in the nature of the progenitors. Here, we present our observations and analysis of GRB 140311A at $z=4.954$. We employ standard cosmological parameters of $\Omega_{m}=0.31, \Omega_{\Lambda}=0.69$, and $H_{0}=68 \mathrm{~km} \mathrm{~s}^{-1} \mathrm{Mpc}^{-1}$ (Ade et al. 2016); all magnitudes are in the $\mathrm{AB}$ system, all uncertainties are at $1 \sigma$, and all times are in the observer frame, unless otherwise specified.

\section{GRB Properties and Observations}

GRB 140311A was discovered by the Swift (Gehrels et al. 2004) Burst Alert Telescope (BAT, Barthelmy et al. 2005) on 2014 March 11 at 21:05:16 UT (Racusin et al. 2014). The burst duration is $T_{90}=71.4 \pm 9.5 \mathrm{~s}$, with a fluence of $F_{\gamma}=$ $(2.3 \pm 0.3) \times 10^{-6} \mathrm{erg} \mathrm{cm}^{-2}(15-150 \mathrm{keV}, 90 \%$ confidence; Krimm et al. 2014). The optical afterglow, discovered by the $1 \mathrm{~m}$ Nanshan telescope at the Xinjiang Observatory (Xu et al. 2014b), was subsequently observed with several other telescopes (D'Avanzo et al. 2014b; Klotz et al. 2014; Xu et al. 2014a; Yoshida et al. 2014). Spectroscopic observations $11.3 \mathrm{hr}$ after the burst with the Gemini-South $8 \mathrm{~m}$ telescope provided a redshift of $z=4.95$ (Tanvir et al. 2014), which was confirmed by the Nordic Optical Telescope (D'Avanzo et al. 2014a).

At this redshift, the Swift/BAT $\gamma$-ray fluence corresponds to an isotropic energy release of $E_{\gamma \text {,iso }}=(1.0 \pm 0.1) \times 10^{53} \mathrm{erg}$ (89-890 keV, rest frame). In the absence of observations by a wide-band $\gamma$-ray satellite and the consequent lack of information about the $\gamma$-ray spectrum, we adopt a K-correction to the rest-frame $1-10^{4} \mathrm{keV}$ band of a factor of $2.7 \pm 0.9$ to determine $E_{\gamma, \text { iso }} \approx(2.7 \pm 0.9) \times 10^{53} \mathrm{erg}$, where the uncertainty is dominated by the uncertainty in the K-correction (LBT14).

\subsection{X-Ray: Swift/XRT}

Swift X-ray Telescope (XRT, Burrows et al. 2005) observations of GRB 140311A were delayed due to an Earth limb constraint and began 0.11 days after the BAT trigger. The X-ray afterglow was localized to R.A. $=13^{\mathrm{h}} 57^{\mathrm{m}} 13^{\mathrm{s}} .25$, decl. $=+00^{\circ} 38^{\prime} 30^{\prime \prime} 8$ (J2000), with an uncertainty radius of $1 . .5$ (90\% containment). ${ }^{9}$ XRT continued observing the afterglow for 4.7 days in photon counting mode. We extract XRT PC-mode spectra using the online tool on the Swift website (Evans et al. 2007, 2009). ${ }^{10}$ We downloaded the event and response files generated by the online tool in these time bins, and fit them using the HEASOFT (v6.19) software package and corresponding calibration files. We used Xspec to fit all available PC-mode data, assuming a photoelectrically absorbed power-law model (tbabs $\times$ ztbabs $\times$ pow) and fixing the galactic absorption column to $N_{\mathrm{H}, \mathrm{Gal}}=$ $2.81 \times 10^{20} \mathrm{~cm}^{-2}$ (Willingale et al. 2013). We find no evidence for excess absorption in the host galaxy, and therefore freeze the intrinsic absorption column to $N_{\mathrm{H} \text {,int }}=0$ in the fit. The parameters of our best-fit spectral model are listed in Table 1. In the following analysis, we take the $0.3-10 \mathrm{keV}$ count rate light curve from the Swift website and compute the $1 \mathrm{keV}$ flux density using our spectral model. We combine the uncertainty in flux calibration based on our spectral analysis

\footnotetext{
9 http://www.swift.ac.uk/xrt_positions/00591390/

${ }^{10}$ http://www.swift.ac.uk/xrt_spectra/00591390/
}

Table 1

XRT Spectral Analysis for GRB 140311A

\begin{tabular}{lc}
\hline \hline Parameter & Value \\
\hline$T_{\text {start }}(\mathrm{s})$ & $9.5 \times 10^{3}$ \\
$T_{\text {end }}(\mathrm{s})$ & $6.3 \times 10^{5}$ \\
$N_{\mathrm{H}, \mathrm{gal}}\left(10^{20} \mathrm{~cm}^{-2}\right)$ & 2.81 \\
$N_{\mathrm{H}, \text { int }}\left(10^{22} \mathrm{~cm}^{-2}\right)$ & $<3.9^{\mathrm{a}}$ \\
Photon index, $\Gamma$ & $1.66 \pm 0.08$ \\
Flux $\left(0.3-10 \mathrm{keV}\right.$, observed; erg cm $\left.\mathrm{er}^{-2} \mathrm{~s}^{-1}\right)$ & $2.0 \times 10^{-12}$ \\
Flux $\left(0.3-10 \mathrm{keV}\right.$, unabsorbed; $\left.\mathrm{erg} \mathrm{cm}^{-2} \mathrm{~s}^{-1}\right)$ & $2.1 \times 10^{-12}$ \\
Counts to flux (observed; erg cm $\left.\mathrm{ct}^{-1}\right)$ & $4.5 \times 10^{-11}$ \\
Counts to flux (unabsorbed; $\left.\mathrm{erg} \mathrm{cm}^{-2} \mathrm{ct}^{-1}\right)$ & $4.4 \times 10^{-11}$ \\
C statistic (dof) & $193(214)$ \\
\hline
\end{tabular}

Note.

a $3 \sigma$ upper limit.

Table 2

Swift/UVOT Observations of GRB 140311A

\begin{tabular}{lcc}
\hline \hline $\begin{array}{l}\Delta t \\
(\text { days })\end{array}$ & Filter & $\begin{array}{c}3 \sigma \text { Flux Upper Limit } \\
(\mathrm{mJy})\end{array}$ \\
\hline $1.16 \times 10^{-1}$ & & $3.6 \times 10^{-3}$ \\
$4.52 \times 10^{-1}$ & white & $4.6 \times 10^{-3}$ \\
$4.43 \times 10^{-1}$ & white & $1.6 \times 10^{-2}$ \\
\hline
\end{tabular}

(This table is available in its entirety in machine-readable form.)

$(6 \%)$ in quadrature with the statistical uncertainty from the online light curve.

\subsection{Optical}

The Swift UV/Optical Telescope (UVOT; Roming et al. 2005) observed GRB 140311A beginning 0.11 days after the burst (Holland \& Racusin 2014). We analyzed the UVOT data using HEASOFT (v. 6.19) and corresponding calibration files and list our derived upper limits in Table 2.

We further analyze $i^{\prime}$ - and $r^{\prime}$-band acquisition images of the field taken at Gemini-North and Gemini-South, respectively. ${ }^{11}$ We downloaded the images and performed photometry in a 2 . 5 aperture calibrated to SDSS. We present the results and a compilation of all other optical observations reported in GCN circulars in Table 3.

\subsection{Optical Spectroscopy}

We obtained a single epoch of optical spectroscopy of the afterglow using the Gemini Multi-Object Spectrograph (GMOS; Hook et al. 2004) on the $8 \mathrm{~m}$ Gemini-North telescope through proposal GS-2014A-Q-36 (PI: Berger). A dithered pair of $900 \mathrm{~s}$ exposures was taken at a midpoint of 13:11 UT on 2014 March 12 ( 0.67 days after the burst) using a $1^{\prime \prime}$ slit, the R831 grating, and an OG515 order-blocking filter. Our setup covered the wavelength range 6945-9070 $\AA$ with a resolution of $\sim 2.9 \AA$ (full width at half maximum). We applied standard data analysis tasks using a combination of IRAF $^{12}$ and custom IDL scripts to provide a flux calibration and telluric correction based on observations of archival standard stars. We normalized the

\footnotetext{
11 https://archive.gemini.edu

12 IRAF is distributed by the National Optical Astronomy Observatories, which are operated by the Association of Universities for Research in Astronomy, Inc., under cooperative agreement with the National Science Foundation.
} 
Table 3

Optical Observations of GRB 140311A

\begin{tabular}{|c|c|c|c|c|c|c|c|c|}
\hline $\begin{array}{l}\Delta t \\
\text { (days) }\end{array}$ & Observatory & $\begin{array}{c}\text { Instrument/ } \\
\text { Telescope }\end{array}$ & Filter & $\begin{array}{l}\text { Frequency } \\
(\mathrm{Hz})\end{array}$ & $\begin{array}{l}\text { Flux Density } \\
(\mathrm{mJy})\end{array}$ & $\begin{array}{l}\text { Uncertainty }^{\mathrm{a}} \\
(\mathrm{mJy})\end{array}$ & $\begin{array}{c}\text { Detection? } \\
1=\text { Yes }\end{array}$ & GCN \\
\hline$\overline{1.16 \times 10^{-4}}$ & Blagoveschensk & MASTER & $C R$ & $4.56 \times 10^{14}$ & 8.28 & 2.76 & 0 & GCN 15946 \\
\hline $3.13 \times 10^{-4}$ & Tunka & MASTER & $C R$ & $4.56 \times 10^{14}$ & 1.02 & $3.41 \times 10^{-1}$ & 0 & GCN 15946 \\
\hline $1.47 \times 10^{-3}$ & Tunka & MASTER & $C R$ & $4.56 \times 10^{14}$ & 1.02 & $3.41 \times 10^{-1}$ & 0 & GCN 15946 \\
\hline $3.76 \times 10^{-3}$ & Gingin & Zadko & $R$ & $4.56 \times 10^{14}$ & $3.61 \times 10^{-2}$ & $1.15 \times 10^{-2}$ & 1 & GCN 15952 \\
\hline
\end{tabular}

Note. $C R$ indicates clear filter calibrated to $R$-band.

${ }^{a}$ An uncertainty of $0.2 \mathrm{AB}$ mag is assumed where not provided. All upper limits are $3 \sigma$. The data have not been corrected for Galactic extinction.

(This table is available in its entirety in machine-readable form.)

Table 4

Absorption Lines in the GRB 140311A Spectrum

\begin{tabular}{|c|c|c|c|c|c|}
\hline$\lambda_{\text {obs }}(\AA)^{\mathrm{a}}$ & Line ID & $\lambda_{\text {rest }}(\AA)$ & Redshift & $\mathrm{EW}(\AA)^{\mathrm{b}}$ & $\log \left(N_{\mathrm{X}} / \mathrm{cm}^{-2}\right)^{\mathrm{c}}$ \\
\hline $7503.55 \pm 0.48$ & Si II & 1260.42 & $4.9532 \pm 0.0004$ & $0.85 \pm 0.21$ & $13.78 \pm 0.11$ \\
\hline $7530.28 \pm 0.78$ & $\mathrm{Si} \mathrm{II}^{*}$ & 1264.74 & $4.9540 \pm 0.0006$ & $0.69 \pm 0.26$ & $13.73 \pm 0.17$ \\
\hline $7753.71 \pm 0.29$ & O I & 1302.17 & $4.9545 \pm 0.0002$ & $0.34 \pm 0.14$ & $14.67 \pm 0.18$ \\
\hline $7765.95 \pm 0.28$ & Si II & 1304.37 & $4.9538 \pm 0.0002$ & $0.35 \pm 0.08$ & $14.39 \pm 0.10$ \\
\hline $7769.02 \pm 0.24$ & $\mathrm{OI}^{*}$ & 1304.86 & $4.9539 \pm 0.0002$ & $0.41 \pm 0.09$ & $14.74 \pm 0.09$ \\
\hline $7775.69 \pm 0.31$ & unidentified & $\ldots$ & $\ldots$ & $2.40 \pm 0.74^{\mathrm{d}}$ & $\ldots$ \\
\hline $7795.77 \pm 0.20$ & Si II* & 1309.28 & $4.9543 \pm 0.0002$ & $0.32 \pm 0.11$ & $14.16 \pm 0.15$ \\
\hline $7945.71 \pm 0.25$ & C II & 1334.53 & $4.9539 \pm 0.0002$ & $0.69 \pm 0.12$ & $14.54 \pm 0.08$ \\
\hline $7953.23 \pm 0.28$ & C II* & 1335.71 & $4.9543 \pm 0.0002$ & $0.57 \pm 0.12$ & $14.50 \pm 0.09$ \\
\hline $8296.77 \pm 0.35$ & Si IV & 1393.76 & $4.9528 \pm 0.0003$ & $1.46 \pm 0.16$ & $14.21 \pm 0.05$ \\
\hline $8351.96 \pm 0.37$ & Si IV & 1402.77 & $4.9539 \pm 0.0003$ & $1.41 \pm 0.15$ & $14.49 \pm 0.05$ \\
\hline
\end{tabular}

Notes.

a Vacuum wavelengths.

${ }^{b}$ Rest frame.

${ }^{c}$ Lower limit due to optically thin assumption.

${ }^{\mathrm{d}} E W$ for the unidentified line is in the observer frame.

continuum by fitting a power law at long wavelengths $(\lambda>8100 \AA)$, excluding strong absorption features. The final combined spectrum has a median signal-to-noise ratio per resolution element of $\sim 12$ in the continuum (Figure 1).

The spectrum exhibits a broad damped Ly $\alpha$ absorber (DLA) centered near $7200 \AA$, as well as several narrow absorption lines redward of Ly $\alpha$. We fit individual Gaussian profiles to each absorption line except in cases of blends, where double Gaussian profiles are used. We report the line identifications and equivalent widths (EW) in Table 4, along with inferred column densities using atomic data collected by Prochaska et al. (2007). Since most of the lines are saturated, our derived column densities represent only lower limits. We take a weighted average of the narrower lines and derive a mean redshift $z=4.9540$. The presence of excited fine-structure transitions from $\mathrm{C} \mathrm{II}^{*}, \mathrm{OI}^{*}$, and $\mathrm{Si} \mathrm{II}^{*}$ in this system mark this as the redshift of the GRB. We fit a DLA model to the continuum in the range 7200-8200 $\AA$, excluding absorption lines and a region of strong telluric absorption. We fix the redshift of the absorber to match the narrow lines and find $\log \left(N_{\mathrm{HI}} / \mathrm{cm}^{-2}\right) \approx 22.2$ for the host DLA, consistent with the upper limit on $N_{\mathrm{H} \text {,int }}$ derived from the X-ray afterglow (Table 1).

\subsection{Millimeter: CARMA}

We observed GRB 140311A with the Combined Array for Research in Millimeter Astronomy (CARMA) beginning on 2014 March 13.32 UT (1.66 days after the burst; PI: Zauderer) in continuum wide-band mode with $8 \mathrm{GHz}$ bandwidth $(16$ windows, $487.5 \mathrm{MHz}$ each) at a mean frequency of $85.5 \mathrm{GHz}$. Following an initial detection, we obtained two additional epochs. All observations utilized J1337-139 as phase calibrator, 3C279 as bandpass calibrator and Mars as flux calibrator. We derived a linelength calibration to account for thermal changes in the delays through the optical fibers connecting the CARMA antennas to the correlator using MIRIAD (Sault et al. 1995), and performed the rest of the data analysis using the Common Astronomy Software Applications (CASA; McMullin et al. 2007). We summarize our millimeter-band observations in Table 5.

\subsection{Centimeter: VLA}

We observed the afterglow using the Karl G. Jansky Very Large Array (VLA) starting 2.48 days after the burst through program 14A-344 (PI: Berger). We detected and tracked the flux density of the afterglow from $1.2 \mathrm{GHz}$ to $37 \mathrm{GHz}$ over six 


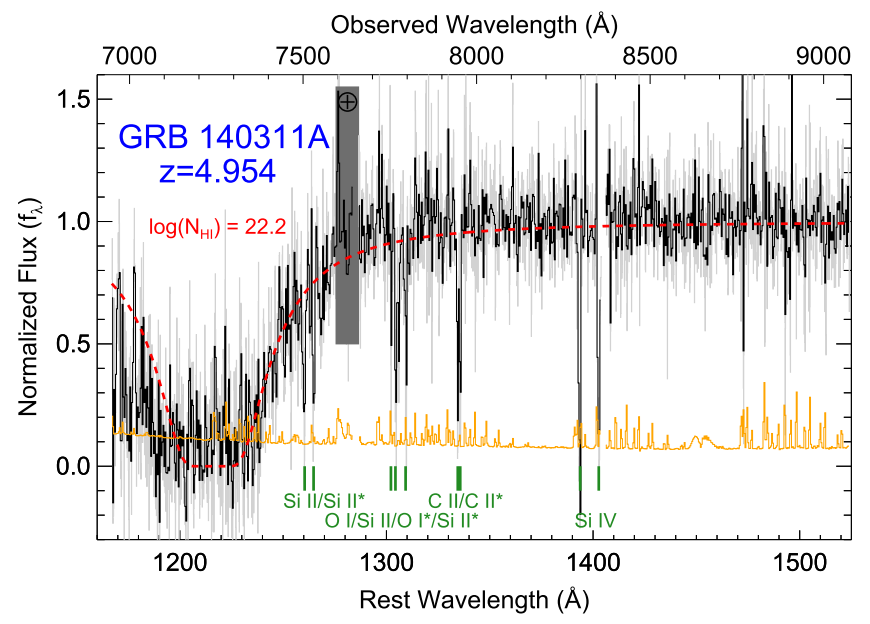

Figure 1. Gemini spectrum of the afterglow of GRB 140311A. The black spectrum has been binned for display purposes only, and the original spectrum is shown as gray in the background. Gaps in the data reflect the GMOS-N CCD chip gaps and the region most adversely affected by telluric absorption is marked with the dark gray box. The formal $1 \sigma$ uncertainty for the binned spectrum is shown in orange. Absorption lines from the host galaxy are identified in green and a fit to a host DLA model is shown as the red dashed line.

Table 5

GRB 140311A: Log of Radio Observations

\begin{tabular}{llcccc}
\hline \hline $\begin{array}{l}\Delta t \\
(\text { days })\end{array}$ & Facility & $\begin{array}{c}\text { Frequency } \\
(\mathrm{GHz})\end{array}$ & $\begin{array}{c}\text { Flux Density } \\
(\mu \mathrm{Jy})\end{array}$ & $\begin{array}{c}\text { Uncertainty } \\
(\mu \mathrm{Jy})\end{array}$ & Det.? \\
\hline 1.66 & CARMA & 85.5 & 699 & 184 & 1 \\
2.48 & VLA & 4.9 & 40.1 & 13.4 & 0 \\
2.48 & VLA & 7.0 & 33.6 & 11.2 & 0 \\
2.63 & VLA & 19.2 & 243.0 & 23.0 & 1 \\
2.63 & VLA & 24.5 & 259.0 & 29.0 & 1 \\
\hline
\end{tabular}

Note. The last column indicates a detection (1) or non-detection (0). This is a sample of the full table available online.

(This table is available in its entirety in machine-readable form.)

epochs until 82.3 days after the burst, when it faded beyond detection at all frequencies. We used 3C286 as the flux and bandpass calibrator and J1354-0206 as gain calibrator. We carried out data reduction using CASA and list the results of our VLA observations in Table 5.

\section{Basic Considerations}

We now interpret the X-ray, optical, and radio observations in the standard synchrotron framework (Sari et al. 1998; Granot \& Sari 2002) in which the observed spectra are characterized by power-law segments connected at characteristic break frequencies: the self-absorption frequency $\left(\nu_{\mathrm{a}}\right)$, the characteristic synchrotron frequency $\left(\nu_{\mathrm{m}}\right)$, and the cooling frequency $\left(\nu_{\mathrm{c}}\right)$. The electrons responsible for the observed radiation are assumed to form a power-law distribution in energy with index $p$. The parameters of the shock creating the radiation are the total isotropic-equivalent kinetic energy $\left(E_{\mathrm{K}, \text { iso }}\right)$, the circumburst density $\left(n_{0}\right.$ in the case of a constant density environment, or $A_{*}$ in the case of a wind environment), the fraction of shock energy imparted to

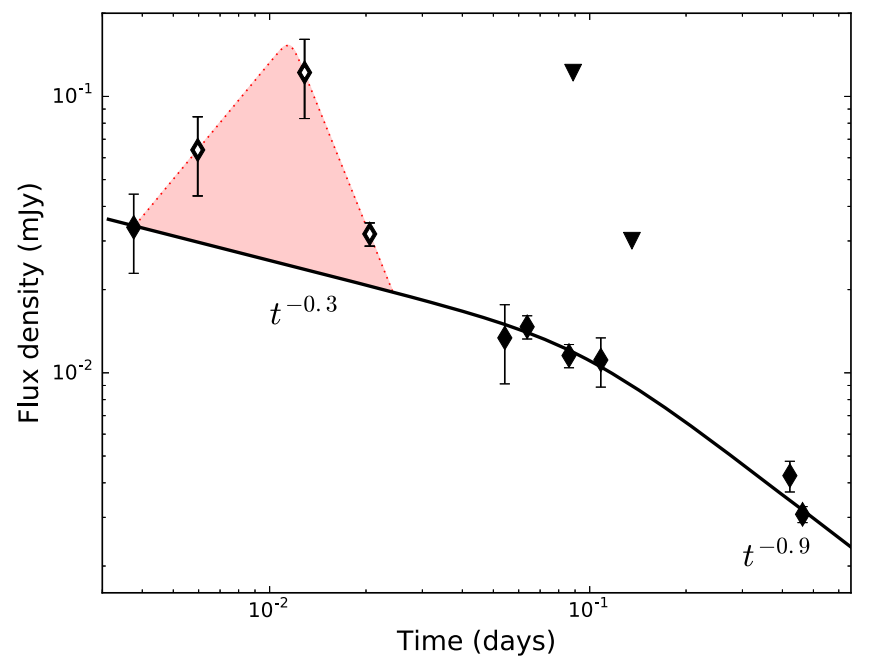

Figure 2. Optical $R$-band light curve of GRB 140311A (black points), together with a broken power-law model (solid). The smoothness of the break has been fixed to $y=5.0$. The light curve exhibits a flare between $\approx 3.8 \times 10^{-3}$ and $\approx 2 \times 10^{-2}$ days (red shaded region), which we exclude from our multiwavelength modeling.

relativistic electrons $\left(\epsilon_{\mathrm{e}}\right)$ and the fraction imparted to magnetic fields $\left(\epsilon_{\mathrm{B}}\right)$.

\subsection{Optical and X-Rays}

The $R$-band light curve ${ }^{13}$ exhibits a rapid rise, with $\alpha_{\mathrm{R}, \mathrm{f} 1}=1.0 \pm 0.1$ from $3.8 \times 10^{-3}$ to $1.3 \times 10^{-2}$ days followed by a steep decline with $\alpha_{\mathrm{R}, \mathrm{f} 2}=-2.8 \pm 0.7$ to $2.0 \times 10^{-2}$ days (Figure 2). Optical flares with rapid rise and decline have previously been observed in GRB afterglows, and cannot be explained under the standard synchrotron framework (e.g., Li et al. 2012). Such flares may be related to continued activity of the central engine (Ghisellini et al. 2009; Nardini et al. 2010), and we therefore do not include the flare in our analysis.

The underlying $R$-band light curve can be fit with a single power law from $3.8 \times 10^{-3}$ days to $1.1 \times 10^{-1}$ days, with $\alpha_{\mathrm{R}, 1}=0.34 \pm 0.06$; however, this fit overpredicts the RATIR $r^{\prime}$ band data at $\approx 0.42$ days by a factor of $\approx 1.5$, suggesting a temporal break before 0.42 days. A broken power-law fit yields a break time of $\approx 0.1$ days, and a post-break decay rate of $\alpha_{\mathrm{R}, 2} \approx-0.9$. In the next section, we show that this break is consistent with the passage of $\nu_{\mathrm{m}}$ through the optical band. We note that the optical point at $3.8 \times 10^{-3}$ days may have a significant contribution from the flare, and thus the pre-break light curve may be shallower than inferred here. In the standard synchrotron framework, such a shallow light curve is naturally explained in the spectral ordering $\nu_{\mathrm{c}}<\nu_{\mathrm{opt}}<\nu_{\mathrm{m}}$, where the light curve declines as $t^{-1 / 4}$. An alternate possibility is that this shallow decline arises from energy injection into the blast wave; however, the resulting model, with a lower value of $\nu_{\mathrm{m}}$ and higher peak flux density, is not consistent with the radio observations described in the next section. The location of $\nu_{\mathrm{c}}<\nu_{\mathrm{opt}}$ is typically only expected at early times or when the circumburst density is large. We show below that a highdensity environment is also demanded by the centimeter-band data, and is the likely cause for a sustained fast cooling

$\overline{13}$ We employ the convention, $F_{\nu} \propto t^{\alpha} \nu^{\beta}$ throughout. 


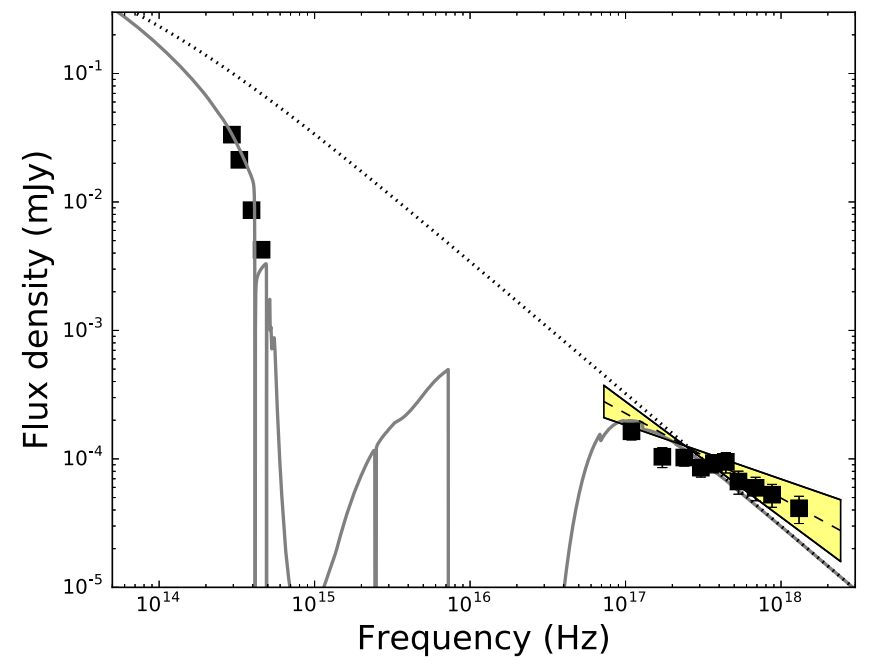

Figure 3. Optical to X-ray spectral energy distribution of the afterglow of GRB 140311A at 0.42 days (black points) together with the best-fit ISM model to the multiwavelength data (gray, solid; see Section 4). The dotted line is the best-fit afterglow model, corrected for extinction and IGM absorption. The optical data are from RATIR (Littlejohns et al. 2014), while the X-ray data have been interpolated to 0.42 days using a broken power-law fit to the Swift XRT light curve (Figure 4$)$. The optical spectrum is steep $(\beta \approx-4)$, partly due to IGM absorption, but also due to dust extinction in the host galaxy (Section 3). The yellow shaded region indicates the $3 \sigma$ error bar for the X-ray spectral index (Table 1).

evolution $\left(\nu_{\mathrm{c}}<\nu_{\mathrm{m}}\right)$. Since $\nu_{\mathrm{c}}$ decreases with time in the ISM environment and increases in the wind environment, a low value of $\nu_{\mathrm{c}}$ at later times is more naturally explained in the ISM case.

The optical spectral index between the RATIR $Y$-band and $r^{\prime}$-band observations at 0.42 days is extremely steep, $\beta_{\text {opt }}=-4.6 \pm 0.1$ (Figure 3). The flux density at $i^{\prime}$ - and $r^{\prime}$ band is expected to be suppressed due to IGM absorption, given $z=4$.954. However, the spectral index between $Y$ - and $z^{\prime}$-bands is also steep $\left(\beta_{\mathrm{Yz}}=-4.0 \pm 1.0\right)$, while the spectral index between the $Y$-band and the $\mathrm{X}$-rays is much shallower $\left(\beta_{\mathrm{opt}, \mathrm{X}}=-0.83 \pm 0.04\right)$. This suggests significant dust extinction along the line of sight through the host galaxy.

The X-ray light curve is well fit with a broken power-law model, with an initial decline rate of $\alpha_{\mathrm{X}, 1}=-1.16 \pm 0.08$ steepening to $\alpha_{\mathrm{X}, 2}=-1.9 \pm 0.4$ at $t_{\mathrm{b}}=1.2 \pm 0.7$ days (Figure 4). For $\nu_{\mathrm{c}}, \nu_{\mathrm{m}}<\nu_{\mathrm{X}}$, the pre-break decline rate indicates $p=2.2 \pm 0.1$ in both the ISM and wind environments. The shallow $\mathrm{X}$-ray spectral index $\left(\beta_{\mathrm{X}}=\right.$ $-0.66 \pm 0.08)$ is in tension with this interpretation, since the latter requires $\beta_{\mathrm{X}} \approx-1.1$. It is possible that KleinNishina suppression of inverse Compton cooling or the contribution of another component, such as inverse Compton radiation, causes the observed flattening of the slope (Sari \& Esin 2001; Lemoine 2015). We note that the late time decline rate of $\alpha_{\mathrm{X}, 2} \approx-2$ is consistent with post-jet break evolution with $p \approx 2$, since $\alpha \approx-p$ at $t>t_{\text {jet }}$.

To summarize, the optical light curve exhibits a shallow decay until $\approx 0.1$ days, suggesting the afterglow SED is in the fast cooling regime with $\nu_{\mathrm{c}}<\nu_{\mathrm{opt}}<\nu_{\mathrm{m}}$ until $\approx 0.1$ days. The $\mathrm{X}$-ray light curve exhibits a steepening at $\approx 1$ days indicative of a jet break. The post-break decline indicates that $p \approx 2$, consistent with the pre-break X-ray light curve for $\nu_{\mathrm{c}}, \nu_{\mathrm{m}}<\nu_{\mathrm{opt}}, \nu_{\mathrm{X}}$.

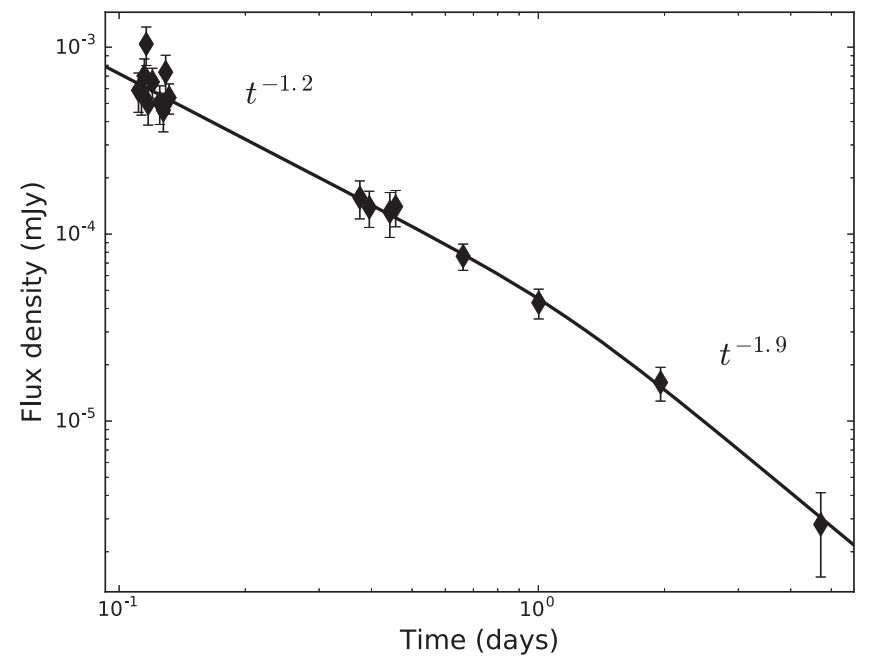

Figure 4. Swift/XRT light curve of GRB 140311A at $1 \mathrm{keV}$ (black points), together with a broken power-law model (solid; Section 3). The smoothness of the break has been fixed to $y=5.0$.

\subsection{Radio}

Synchrotron self-absorption is expected to result in a steep spectral index $(\beta=11 / 8$ to $5 / 2)$ at low frequencies. A measurement of the self-absorption frequency yields a strong constraint on the circumburst density. In our first joint VLA and CARMA observation of the afterglow at $\approx 2.5$ days, we find a steep slope, $\beta_{1}=2.0 \pm 0.3$, from $7 \mathrm{GHz}$ to $24.5 \mathrm{GHz}$, flattening to $\beta_{2}=0.4 \pm 0.1$ at $24.5-85.5 \mathrm{GHz}$ (Figure 5). The radio SED at 4.5 days also exhibits a clear transition from a self-absorbed to an optically thin slope at $\approx 10 \mathrm{GHz}$. To track the evolution of this break frequency, which we identify as $\nu_{\mathrm{a}}$, we fit the radio SEDs between 2.5 and 18.5 days with a broken power-law model described by

$$
F_{\nu}=F_{\mathrm{b}}\left(\frac{\left(\nu / \nu_{\mathrm{b}}\right)^{-y \beta_{1}}+\left(\nu / \nu_{\mathrm{b}}\right)^{-y \beta_{2}}}{2}\right)^{-1 / y},
$$

with $\beta_{1}=2, \beta_{2}=1 / 3$, and smoothness, $y=5 .{ }^{14}$ We present the results in Table 6 . Fitting the evolution of the break frequency and break flux density as power laws with time, we find $\alpha_{\nu}=-0.3 \pm 0.1$ and $\alpha_{\mathrm{F}}=-0.59 \pm 0.05$, which is more consistent with post-jet break evolution $\left(\alpha_{\nu}=-0.2\right.$, $\left.\alpha_{\mathrm{F}}=-0.4\right)$ than a spherical outflow in an ISM $\left(\alpha_{\nu}=0\right.$, $\left.\alpha_{\mathrm{F}}=0.5\right)$ or wind $\left(\alpha_{\nu}=-0.6, \alpha_{\mathrm{F}}=-0.2\right)$ environment.

The observed value of $\nu_{\mathrm{a}}$ and $F_{\nu, \mathrm{a}}$ at 4.5 days results in a moderately high density. To show this, we define ${ }^{15}$ the quantity

$$
\xi_{a} \equiv \nu_{\mathrm{a}, \mathrm{GHz}}^{2} / F_{\nu, \mathrm{a}, \mathrm{mJy}}=46.8 \epsilon_{\mathrm{B}} n_{0} d_{\mathrm{L}, 28}^{2} t_{d}^{-1},
$$

where $d_{\mathrm{L}, 28}=14.5$ is the luminosity distance divided by $10^{28} \mathrm{~cm}$, and $t_{d}$ is the observer time in days. Computing $\xi_{a} \approx 4.5 \times 10^{2}$ at 4.5 days where the radio SED is particularly well constrained, we obtain $n \approx 2 \epsilon_{\mathrm{B}, 0.1}^{-1} \mathrm{~cm}^{-3}$, comparable to the mean density of the Milky Way ISM. This density also predicts a low cooling frequency, $\nu_{\mathrm{c}} \approx 2 \times 10^{11} \epsilon_{\mathrm{B}, 0.1}^{-1 / 2}\left(\eta^{-1}-1\right)^{-1 / 2}(t / 0.1 \text { days })^{-1 / 2} \mathrm{~Hz}$, where $\eta$

\footnotetext{
${ }^{14}$ For the purposes of the fit at 2.5 days, we treat the 4.9 and $7.0 \mathrm{GHz}$ upper limits as $3 \sigma$ detections, with flux densities equal to three times the map rms.

${ }^{15}$ This expression is relevant for fast cooling and an ISM environment.
} 

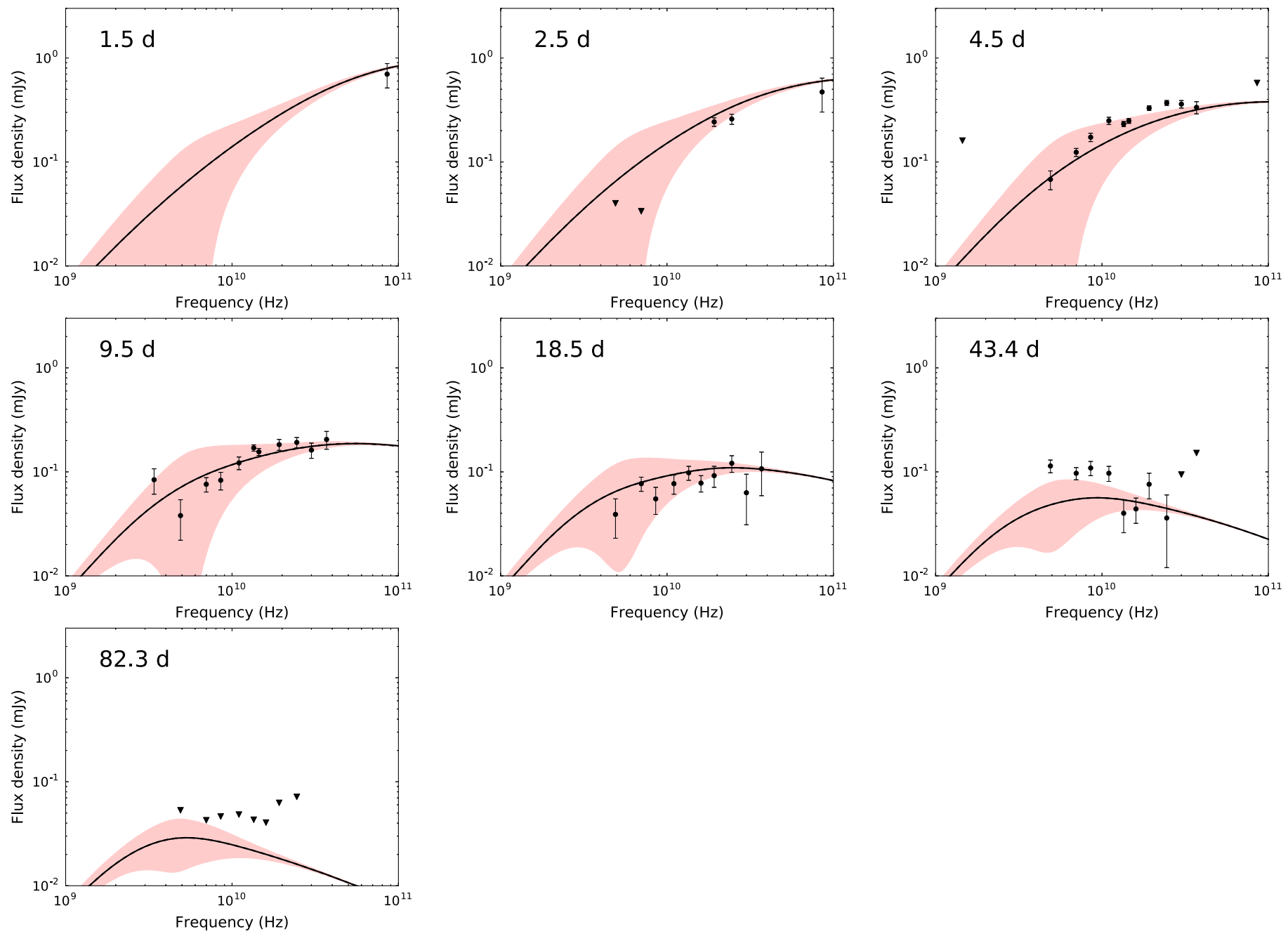

Figure 5. Radio spectral energy distributions of the afterglow of GRB 140311A at multiple epochs starting at 1.5 days, together with the same ISM model in Figure 3. The red shaded regions represent the expected variability due to interstellar scintillation.

Table 6

Radio Spectral Fits for GRB 140311A

\begin{tabular}{lcc}
\hline \hline$\Delta t$ & $\nu_{\text {break }}(\mathrm{GHz})$ & $F_{\text {break }}(\mu \mathrm{Jy})$ \\
\hline 2.5 & $16.7 \pm 2.8$ & $208 \pm 27$ \\
4.5 & $9.6 \pm 0.7$ & $206 \pm 11$ \\
9.5 & $9.9 \pm 1.1$ & $122 \pm 10$ \\
18.5 & $5.7 \pm 1.4$ & $54 \pm 7$ \\
\hline
\end{tabular}

is the radiative efficiency, consistent with the inferred spectral ordering $\nu_{\mathrm{c}}<\nu_{\mathrm{opt}}, \nu_{\mathrm{X}}$ (Section 3.1).

The centimeter-band SED at 43.4 days is inverted, with $\beta=-0.7 \pm 0.2$. This spectral inversion between 18.5 and 43.4 days is only possible if $\nu_{\mathrm{m}}$ crossed the radio band between these two epochs. Taking $\nu_{\mathrm{m}} \approx 5 \mathrm{GHz}$ at 43.4 days and a jet break time of $\approx 1$ days from the $\mathrm{X}$-ray light curve (Section 3.1), we find $\nu_{\mathrm{m}}$ passes through $r^{\prime}$-band at $\approx 0.1$ days. We therefore confirm the steepening in the $r^{\prime}$-band light curve between $\approx 0.1$ and 0.42 days as arising from the passage of $\nu_{\mathrm{m}}$ through the optical band.

To summarize, the radio SEDs allow us to locate both $\nu_{\mathrm{a}}$ and $\nu_{\mathrm{m}}$. The inferred location of $\nu_{\mathrm{m}}$ is consistent with the optical and X-ray light curves, while the observed value of $\nu_{\mathrm{a}}$ is consistent with post-jet break evolution for the duration of the radio observations. We focus in the rest of the paper on the ISM model, and present a wind model in Appendix A.

\section{Multiwavelength Modeling}

Following the considerations outlined in Section 3, we now perform a Markov Chain Monte Carlo analysis to determine the physical parameters of the afterglow. We fit all available photometry (with the exception of the optical flare at $1.3 \times 10^{-2}-2.0 \times 10^{-2}$ days) with a forward shock model using the prescription of Granot \& Sari (2002) with $p, \epsilon_{\mathrm{e}}, \epsilon_{\mathrm{B}}, n_{0}$, $E_{\mathrm{K}, \text { iso }}, t_{\mathrm{jet}}$ and the extinction in the host galaxy $\left(A_{\mathrm{V}}\right)$ as free parameters. The details of our modeling procedure are described in LBT14 and Laskar et al. (2015).

We present the best-fit model in Figure 6 and list the derived parameters and their associated uncertainties in Table 7. The SED remains in the fast cooling phase through the entire duration of the X-ray and optical light curves, and transitions to slow cooling at $\approx 9.8$ days. The spectral ordering at 0.1 days is $\nu_{\mathrm{c}}<\nu_{\mathrm{opt}}<\nu_{\mathrm{m}}<\nu_{\mathrm{X}}$. The model requires a moderately high density of $\approx 8 \mathrm{~cm}^{-3}$, as expected from the discussion in 

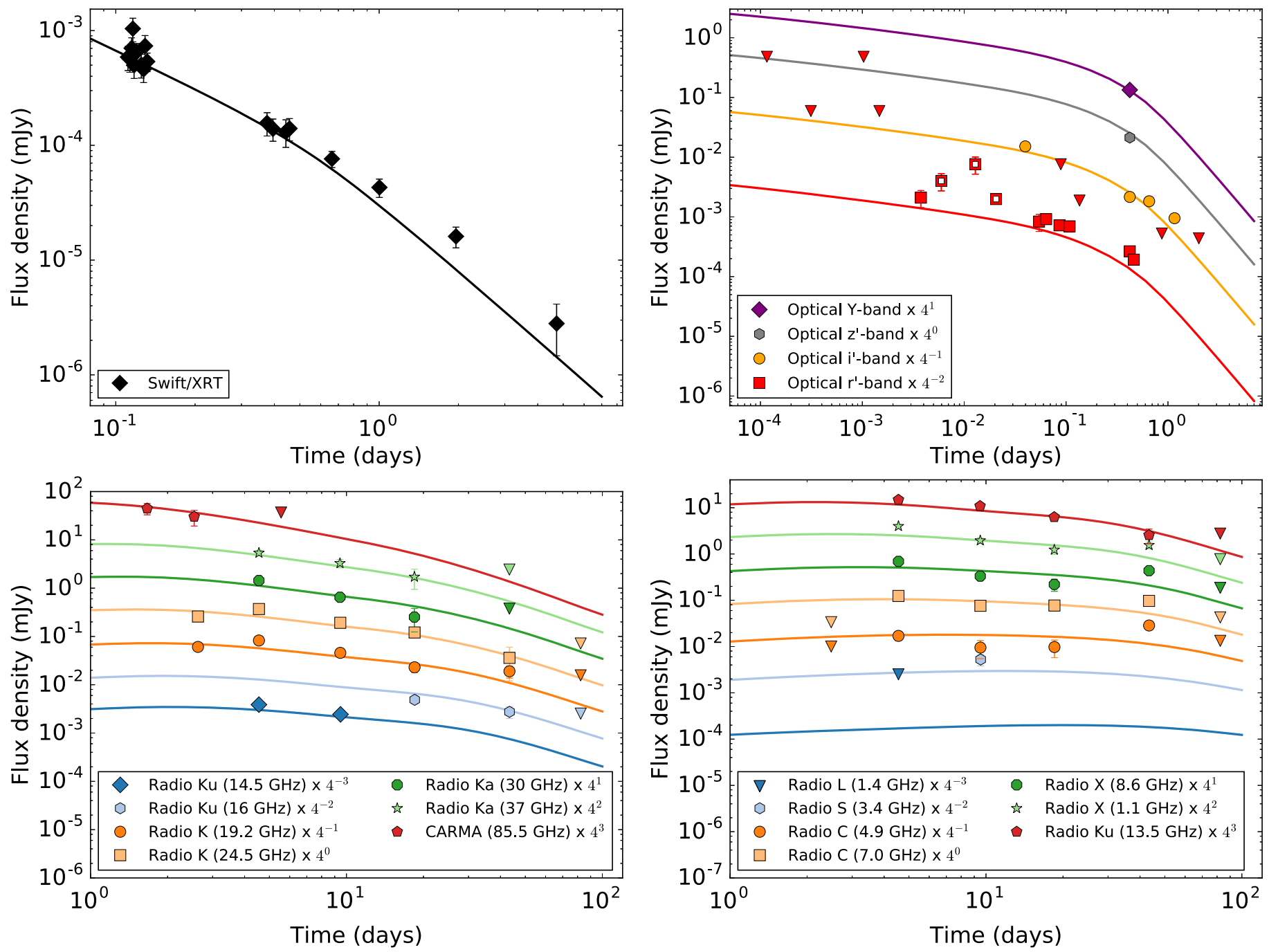

Figure 6. X-ray (top left), optical (top right), and radio (bottom) light curves of the afterglow of GRB 140311A, together with an ISM model (Section 4). The data points with open symbols are not included in the fit. The $r^{\prime}$-band and $i^{\prime}$-band model light curves have been integrated over their corresponding filter response functions, including the effects of IGM absorption (Pei 1992). As the $r^{\prime}$-band is blueward of Ly $\alpha$ at the host redshift, slight discrepancies between the optical $r$-band data and model light curve likely result from deviations in the IGM properties from average along the line of sight to GRB 140311A.

Section 3. The derived modest rest-frame host extinction of $A_{\mathrm{V}} \approx 0.3 \mathrm{mag}$ results in the correct spectral index both within the optical band and from the optical to the $\mathrm{X}$-rays (Figure 3). The jet break time of $\approx 0.6$ days yields an opening angle of $\approx 3^{\circ}$.9. The kinetic energy corrected for beaming is $E_{\mathrm{K}} \approx$ $\left(2.2_{-0.3}^{+0.4}\right) \times 10^{50} \mathrm{erg}$, and the beaming-corrected $\gamma$-ray energy is $E_{\gamma} \approx 7 \times 10^{50} \mathrm{erg}$, yielding a $\gamma$-ray efficiency of $\eta \approx 76 \%$. Histograms of the posterior density for each free parameter are provided in Figure 7, while correlation contours between pairs of the physical parameters $\left(\epsilon_{\mathrm{e}}, \epsilon_{\mathrm{B}}, n_{0}, E_{\mathrm{K} \text {,iso }}\right)$ are presented in Figure 8.

Since the afterglow remains in the fast cooling regime for a long period of time, significant radiative losses may be expected. A simple estimate under the assumption that the light curves can be modeled as produced by a blast wave with decreasing energy (Sari 1997), yields a decrease in the kinetic energy by a factor of $\approx 24$ between the first $R$-band detection at $\approx 3.8 \times 10^{-3}$ days and the jet break at $\approx 0.56$ days. However, our model with constant energy fits the $\mathrm{X}$-ray to radio observations well over this period, suggesting that this prescription for radiative losses may overestimate the effect (Nava et al. 2013). A detailed analysis of this effect requires better sampled optical light curves, as well as allowance for a variation in the Lorentz factor with radius for nonadiabatic blast waves, and is beyond the scope of this work.

The ISM model underpredicts the flux density at $\lesssim 10 \mathrm{GHz}$ at 43.4 days (Figures 5 and 6 ), a feature that is also true of the wind model described in Appendix A. The light curves at $\lesssim 10 \mathrm{GHz}$ rise between 18.5 days and 43.4 days, whereas we expect them to be either flat $\left(F_{\nu<\nu_{\mathrm{a}}} \propto t^{0}\right)$ or slowly declining $\left(F_{\nu_{\mathrm{a}}<\nu<\nu_{\mathrm{m}}} \propto t^{-1 / 3}\right)$. While a transition to nonrelativistic expansion does allow for such a late-time rise in the radio light curve for $\nu_{\mathrm{a}}<\nu<\nu_{\mathrm{m}}$ (Frail et al. 2000), the expected transition to nonrelativistic expansion based on our best-fit model parameters is $t_{\mathrm{NR}} \approx 500$ days (Waxman et al. 1998). One possible explanation for an early transition to nonrelativistic expansion is a late encounter of the blast wave with a density enhancement, which decelerates the outflow rapidly and results in a rebrightening; however, there are no other observations to test this hypothesis. 
Table 7

Parameters for the Best-fit ISM Model

\begin{tabular}{|c|c|c|}
\hline Parameter & Best Fit & MCMC \\
\hline$p$ & 2.06 & $2.08_{-0.01}^{+0.02}$ \\
\hline$\epsilon_{\mathrm{e}}$ & 0.63 & $0.60 \pm 0.10$ \\
\hline$\epsilon_{\mathrm{B}}$ & 0.34 & $0.22_{-0.14}^{+0.23}$ \\
\hline$n_{0}$ & 8.09 & $11.1_{-3.7}^{+9.1}$ \\
\hline$E_{\mathrm{K}, \text { iso }, 52}$ & 8.46 & $8.7_{-1.5}^{+2.5}$ \\
\hline$t_{\text {jet }}$ (days) & 0.56 & $0.57 \pm 0.05$ \\
\hline$\theta_{\text {jet }}(\operatorname{deg})$ & 3.92 & $4.1 \pm 0.3$ \\
\hline$A_{\mathrm{V}}(\mathrm{mag})$ & 0.34 & $0.34 \pm 0.02$ \\
\hline$\nu_{\mathrm{ac}}(\mathrm{Hz})$ & $8.9 \times 10^{8 \mathrm{a}}$ & $\ldots$ \\
\hline$\nu_{\mathrm{sa}}(\mathrm{Hz})$ & $4.1 \times 10^{11}$ & $\ldots$ \\
\hline$\nu_{\mathrm{c}}(\mathrm{Hz})$ & $4.3 \times 10^{11}$ & $\ldots$ \\
\hline$\nu_{\mathrm{m}}(\mathrm{Hz})$ & $9.0 \times 10^{14}$ & $\ldots$ \\
\hline$F_{\nu, \text { peak }}(\mathrm{mJy})$ & 11.3 & $\cdots$ \\
\hline$E_{\gamma}(\mathrm{erg})$ & \multicolumn{2}{|c|}{$\left(6.9_{-2.4}^{+2.6}\right) \times 10^{50}$} \\
\hline$E_{\mathrm{K}}(\mathrm{erg})$ & \multicolumn{2}{|c|}{$\left(2.2_{-0.3}^{+0.4}\right) \times 10^{50}$} \\
\hline$E_{\text {tot }}(\mathrm{erg})$ & \multicolumn{2}{|c|}{$\approx 9 \times 10^{50}$} \\
\hline$\eta_{\mathrm{rad}}$ & \multicolumn{2}{|c|}{$\approx 76 \%$} \\
\hline
\end{tabular}

Note. All break frequencies are listed at 0.1 days.

a This break frequency is not directly constrained by the data.

The model also marginally underpredicts the radio SED between $7 \mathrm{GHz}$ and $37 \mathrm{GHz}$ at 4.5 days. While some of the excess flux may arise from additional processes, such as emission from a reverse shock (RS), we note that there is no clear evidence for RS radiation at any other frequency at any time. On the other hand, the majority of the observed deviation appears to be consistent with the expected contribution of interstellar scintillation; thus there is no compelling evidence for a reverse shock component in the afterglow data for this event.

We note that the ISM model yields a lower flux density for the X-ray light curve after the jet break than observed. Similar late-time X-ray excess emission has been observed in other GRBs, with suggested explanations, including dust echoes and inverse-Compton scattering (Shao \& Dai 2007; Chandra et al. 2008; Liang et al. 2008; Margutti et al. 2010; Fong et al. 2014). Alternatively, the discrepancy may arise from the simplistic treatment of the hydrodynamics at the jet break, such as exponential sideways expansion (Rhoads 1999), which is likely an incomplete description of the blast wave evolution (Zhang \& MacFadyen 2009; Granot \& Piran 2012; Duffell \& Laskar 2017). Interestingly, the wind model for this burst does not suffer from this problem.

\section{Summary and Discussion}

Our multiwavelength model explains the overall behavior of the afterglow over 8 orders of magnitude in frequency and 4 orders of magnitude in time, and indicates $E_{\mathrm{K}} \approx 2 \times 10^{50} \mathrm{erg}$ and $n_{0} \approx 8 \mathrm{~cm}^{-3}$. These values are similar to those obtained for GRBs at $z \sim 1$ (Panaitescu \& Kumar 2002; Yost et al. 2003; Chandra et al. 2008; Cenko et al. 2010, 2011; Laskar et al. 2014), indicating no evolution in these properties with a redshift to $z \sim 5$.

Whereas our best-fit values of the microphysical parameters are high $\left(\epsilon_{\mathrm{e}}+\epsilon_{\mathrm{B}} \approx 1\right)$, we note that there is significant uncertainty in both of these parameters (in particular, the value of $\left.\epsilon_{\mathrm{B}}\right)$. We test this by fixing $\epsilon_{\mathrm{B}} \approx 0.01$, and find that the consequent best-fit model results in a higher cooling frequency, lower peak flux (at $\nu_{\mathrm{c}}$ ) and lower self-absorption frequency, while the resulting millimeter-band flux density is unchanged within the error bars of the CARMA measurements, and the radio observations remain marginally consistent within the expected scatter from ISS. The parameter distributions resulting from these, and all other related families of models, are summarized in our plots of the correlation contours and the posterior density functions. We note that deeper millimeterband observations at higher frequencies than were possible with CARMA (such as at $\approx 200 \mathrm{GHz}$ with ALMA in Band 6) would break some of these degeneracies, reducing the uncertainty in the physical parameters (Figure 9).

We note that the progenitor population of GRBs is also believed to produce type $\mathrm{Ib} / \mathrm{c}$ supernovae, and whenever the latter are detected at radio wavelengths, the inferred density profile is consistent with a wind-like environment (e.g., Chevalier 1998; Berger et al. 2002; Chevalier \& Fransson 2006; Chakraborti et al. 2015). However, we find that an ISM model is a better fit than a wind environment for this burst, as the latter overpredicts the radio emission before 2.5 days. Whereas multiwavelength studies of $z \sim 1$ events have found no preference for either kind of density profile (but see also Schulze et al. 2011), we previously inferred constant-density environments for all GRBs at $z \gtrsim 6$ with radio detections (LBT14). Increasing the sample of $z \gtrsim 5$ GRBs beyond the current set of four events is critical for exploring the statistical significance of this result. We add that millimeter-band observations have the strongest diagnostic power in distinguishing between constant density and wind-like environments, owing to scintillation effects at the centimeter bands (e.g., Yost et al. 2003; Laskar et al. 2015). Thus millimeterband observations at facilities such as ALMA in conjunction with centimeter-band observations at the VLA will play an important role in answering the question of the circumburst density profile and the mass-loss rates of GRB progenitors in the the last moments before core collapse.

Our inferred jet opening angle of $\theta_{\text {jet }} \approx 4^{\circ}$ for this burst is narrower than the median value of $\theta_{\text {jet }}=7.4_{-6.6}^{+11} \quad(95 \%$ confidence interval) for GRBs at $z \sim 1$, but similar to the mean value for GRBs at $z \gtrsim 6\left(\theta_{\text {jet }}=3.6 \pm 0.7\right.$; LBT14), which may indicate that higher redshift GRBs are more strongly collimated. Whereas this interval formally includes the measurements of $\theta_{\text {jet }}$ for the high-redshift sample, we note that the observed values of $\theta_{\text {jet }}$ for the high-redshift sample are all smaller than the best estimate for the median of the comparison sample, similar to the result of LBT14. Our bestfit wind model also yields a narrow opening angle and a high density, and therefore these results are robust to the choice of circumburst density profile. We note that a selection effect arising from the trigger criteria for $\gamma$-ray telescopes, which may select more tightly beamed events at higher redshift owing to the sensitivity threshold of the detectors, remains a possibility. The observed $\gamma$-ray fluence of this event and all the bursts studied in LBT14 is within one standard deviation of the mean for lower-redshift events (Margutti et al. 2013), with no systematic trend toward lower fluences; however, two out of four $z \gtrsim 5$ events, GRB 140311A and 050904, were located through BAT image triggers, which traditionally find lower luminosity events (Lien et al. 2016). We therefore 

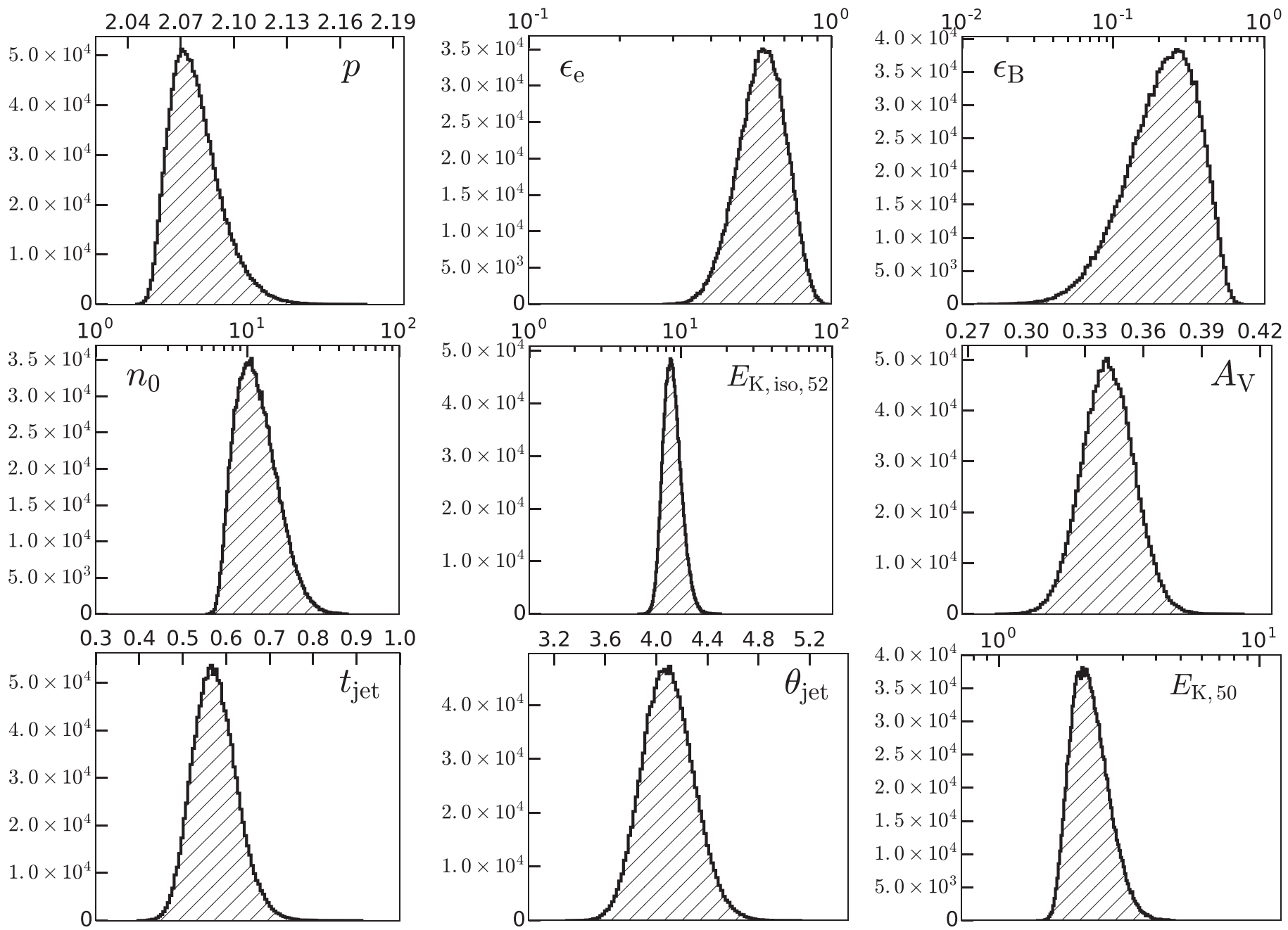

Figure 7. Marginalized posterior probability density functions of the FS parameters from MCMC simulations. We have used the constraint $\epsilon_{\mathrm{e}}+\epsilon_{\mathrm{B}}<1$.

caution that detailed statistical studies should account for possible selection biases due to the criteria used to discover the event.

Our observations afford no compelling evidence for emission from a reverse shock. We have previously found strong RS signatures only in low density environments, which we attribute to the slow cooling RS SEDs expected in such environments (Laskar et al. 2013, 2016; Perley et al. 2014; Alexander et al. 2017). Our best-fit model results in an afterglow SED in the fast cooling regime, increasing the likelihood that the RS SED is fast cooling as well. We speculate that the high density observed in the case of GRB 140311A may suppress RS emission, and that absence of RS signatures may not, therefore, be a signature of highly magnetized ejecta (Uhm et al. 2012).

\section{Conclusions}

Our observations of GRB 140311A are the most detailed joint radio and millimeter observations of a GRB at $z \gtrsim 5$ to date. They reveal an afterglow with parameters typical of events at $z \sim 1$, with the exception of the opening angle, which is narrower, but similar to the values derived for events at $z \gtrsim 6$ and consistent with the hypothesis that GRBs detected at high redshift may be more tightly beamed (LBT14). We find no evidence for a strong reverse shock, and note that synchrotron cooling may suppress the RS emission in this case. Upcoming papers in this series will address the connection between the circumburst density and the likelihood of observing RS emission, as well as the redshift evolution of the opening angles of GRB jets.

We thank the anonymous referee for helpful comments on the manuscript. T.L. is a Jansky Fellow of the National Radio Astronomy Observatory. The Berger Time-Domain Group at Harvard is supported in part by the NSF under grant AST-1411763 and by NASA under grant NNX15AE50G. B.A.Z. acknowledges support from NSF AST-1302954. R.C. acknowledges support from NASA Swift grant NNX16AB04G. VLA observations for this study were obtained via project 14A344. The National Radio Astronomy Observatory is a facility of the National Science Foundation operated under cooperative agreement by Associated Universities, Inc. This research has made use of data supplied by the UK Swift Science Data Centre at the University of Leicester, and of data obtained through the High Energy Astrophysics Science Archive Research Center On-line Service, provided by the NASA/Goddard Space Flight Center. 

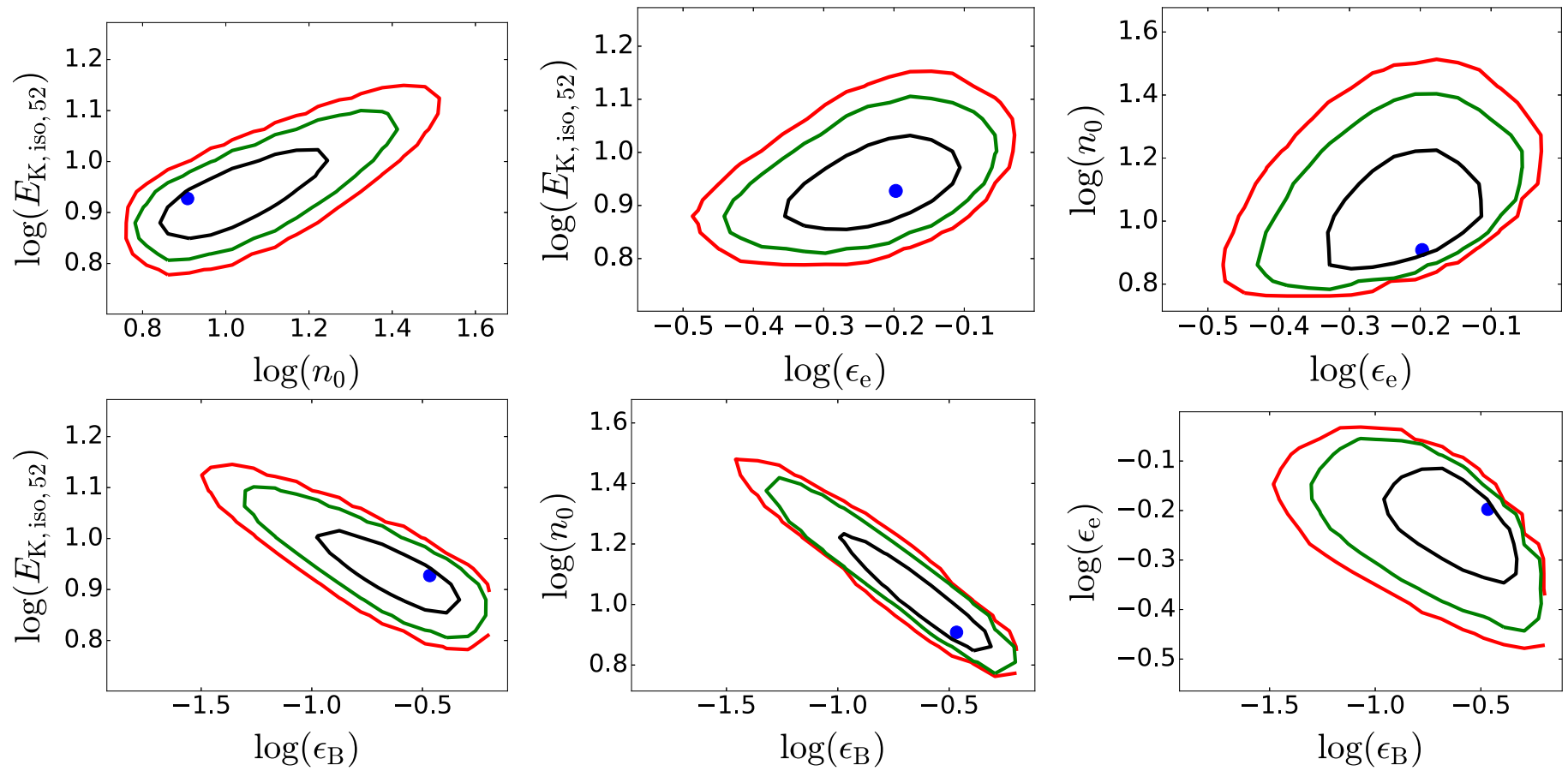

Figure 8. $1 \sigma$ (red), $2 \sigma$ (green), and $3 \sigma$ (black) contours for correlations between the physical parameters $E_{\mathrm{K}, \text { iso }}, n_{0}, \epsilon_{\mathrm{e}}$, and $\epsilon_{\mathrm{B}}$ from Monte Carlo simulations, together with the best-fit model (blue dot). We have used the constraint $\epsilon_{\mathrm{e}}+\epsilon_{\mathrm{B}}<1$.

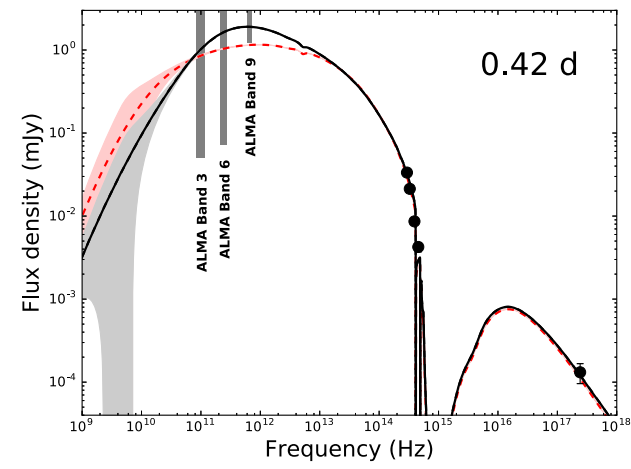

Figure 9. Optical to X-ray spectral energy distribution of the afterglow of GRB 140311A with the best-fit ISM model (black; solid), together with an $\epsilon_{\mathrm{B}} \approx 0.01$ model (red; dashed) for comparison. The shaded regions reflect the $1 \sigma$ uncertainty due to scintillation along the line of sight (the gray band largely overlaps the red band), while the vertical bands indicate the $3 \sigma$ sensitivity of ALMA in three different observing bands with 30 minutes on source.

\section{Appendix A \\ A Wind Model}

We carried out an MCMC analysis for the wind environment similar to the analysis for the ISM case described in Section 4. The parameters of our best-fit model and the results of the MCMC analysis are listed in Table 8. Light curves and radio SEDs are presented in Figures 10 and 11, and histograms of the posterior density and correlation contours between the physical parameters are presented in Figures 12 and 13.

Our best-fit model reproduces the X-ray and optical light curves well, but overpredicts the radio SED in the first two epochs. The spectral break frequencies are in the order $\nu_{\mathrm{c}}<\nu_{\mathrm{a}}<\nu_{\mathrm{m}}$ at 0.1 days, a scenario that occurs more
Table 8

Parameters for the Best-fit Wind Model

\begin{tabular}{|c|c|c|}
\hline Parameter & Best Fit & MCMC \\
\hline$p$ & 2.06 & $2.07_{-0.02}^{+0.03}$ \\
\hline$\epsilon_{\mathrm{e}}$ & 0.50 & $0.49_{-0.15}^{+0.20}$ \\
\hline$\epsilon_{\mathrm{B}}$ & 0.17 & $\left(9.7_{-7.8}^{+20.2}\right) \times 10^{-2}$ \\
\hline$A_{*}$ & 0.23 & $0.29_{-0.10}^{+0.20}$ \\
\hline$E_{\mathrm{K}, \text { iso }, 52}$ & 11.0 & $12.5_{-3.0}^{+8.6}$ \\
\hline$t_{\text {jet }}$ (days) & 1.05 & $1.1_{-0.3}^{+0.4} 5$ \\
\hline$\theta_{\text {jet }}(\operatorname{deg})$ & 2.84 & $2.9 \pm 0.2$ \\
\hline$A_{\mathrm{V}}(\mathrm{mag})$ & 0.39 & $0.40 \pm 0.02$ \\
\hline$\nu_{\mathrm{ac}}(\mathrm{Hz})$ & $8.6 \times 10^{8 a}$ & $\cdots$ \\
\hline$\nu_{\mathrm{c}}(\mathrm{Hz})$ & $8.9 \times 10^{10}$ & $\ldots$ \\
\hline$\nu_{\mathrm{sa}}(\mathrm{Hz})$ & $3.7 \times 10^{11}$ & $\cdots$ \\
\hline$\nu_{\mathrm{m}}(\mathrm{Hz})$ & $4.8 \times 10^{14}$ & $\ldots$ \\
\hline$F_{\nu, \text { peak }}(\mathrm{mJy})$ & 14.5 & $\cdots$ \\
\hline$E_{\gamma}(\mathrm{erg})$ & & $\left(3.4_{-1.2}^{+1.3}\right) \times 10^{50}$ \\
\hline$E_{\mathrm{K}}(\mathrm{erg})$ & & $\left(1.6_{-0.5}^{+1.3}\right) \times 10^{50}$ \\
\hline$E_{\mathrm{tot}}(\mathrm{erg})$ & & $\approx 5 \times 10^{50}$ \\
\hline$\eta_{\mathrm{rad}}$ & & $\approx 68 \%$ \\
\hline
\end{tabular}

Note. All break frequencies are listed at 0.1 days.

${ }^{\mathrm{a}}$ This break frequency is not directly constrained by the data.

frequently in wind environments due to the higher density at small radii (Kobayashi et al. 2004). We note that in this scenario, synchrotron self-absorption prevents the electrons from cooling efficiently and redistributes the electron energy, thus changing the underlying distribution function, an effect that has not been modeled in detail. Accurately fitting the radio SEDs then requires an additional source of opacity in the radio and millimeter-bands, which must disappear by 9.5 days. We 

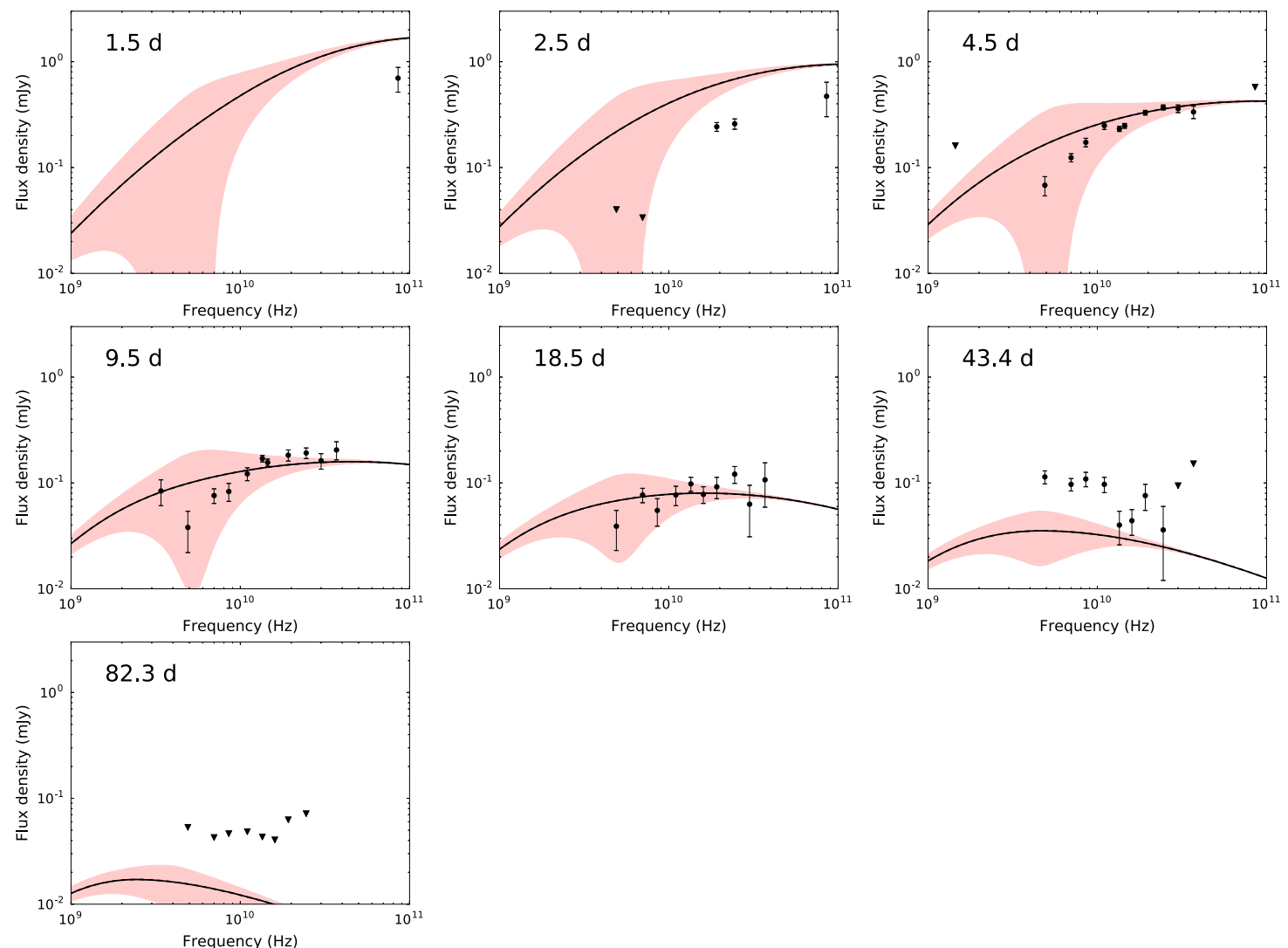

Figure 10. Same as Figure 5, but for a wind environment. The model overpredicts the millimeter and radio observations at 1.5 days and 2.5 days, requiring an additional source of opacity at low frequencies.
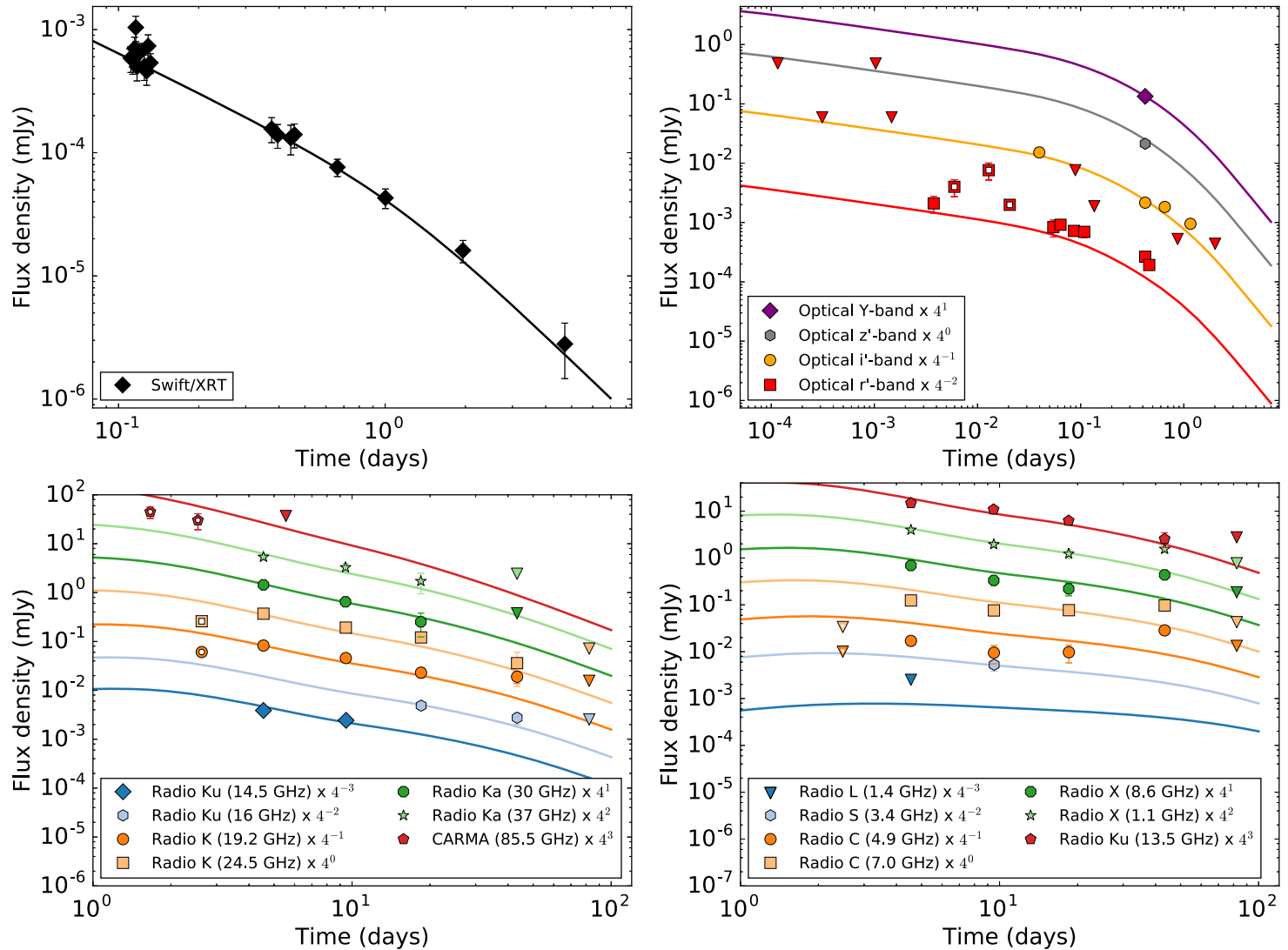

Figure 11. Same as Figure 6, but for a wind environment. The model fits the X-ray and optical light curves well, but overpredicts the radio and millimeter observations at 1.5 days and 2.5 days (Figure 10 ). The data points with open symbols are not included in the multiwavelength fit. 

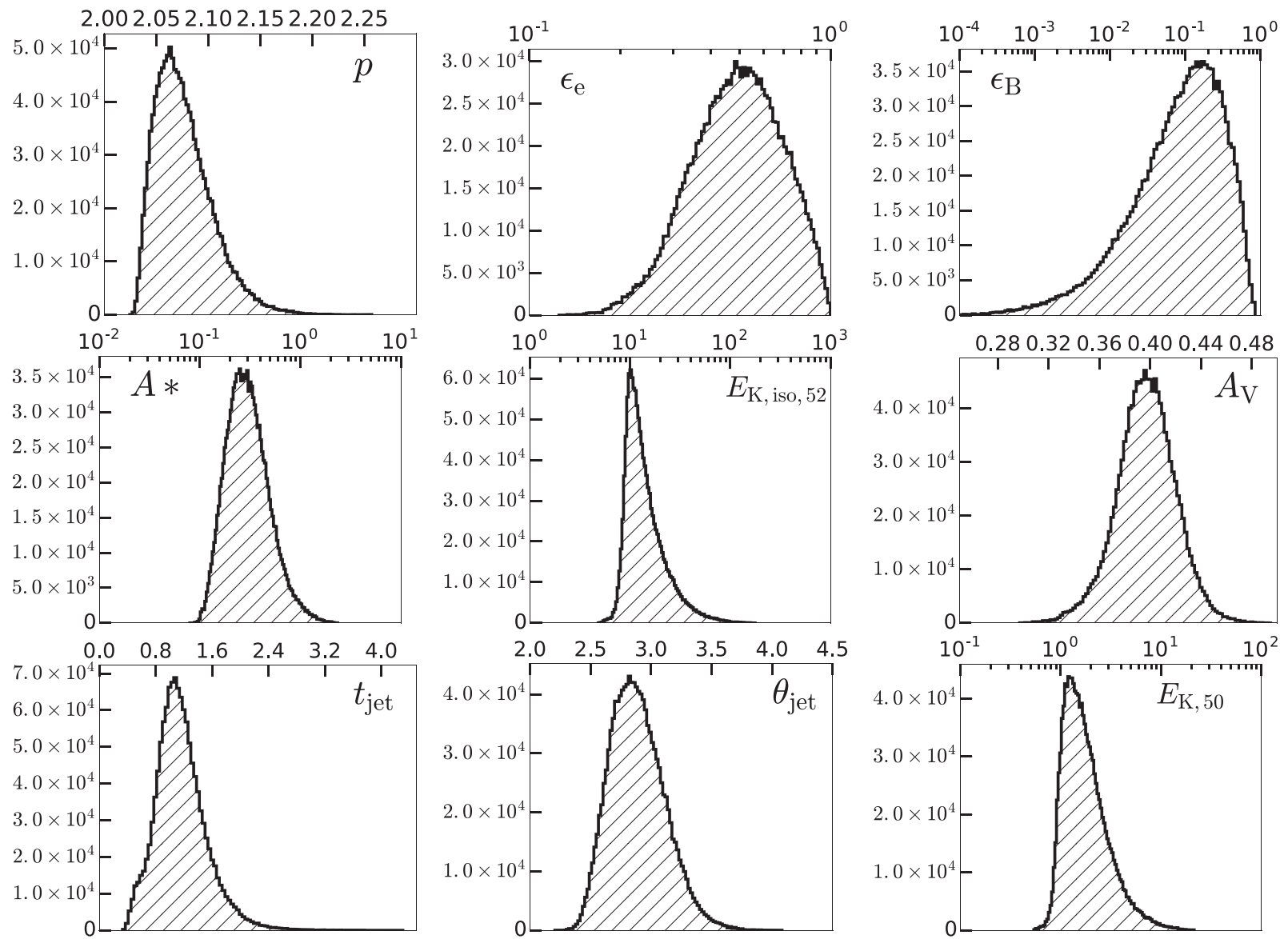

Figure 12. Marginalized posterior probability density functions of the FS parameters from MCMC simulations for a wind environment. We have restricted $\epsilon_{\mathrm{e}}+\epsilon_{\mathrm{B}}<1$, and do not include the radio data before 2.5 days in the analysis.
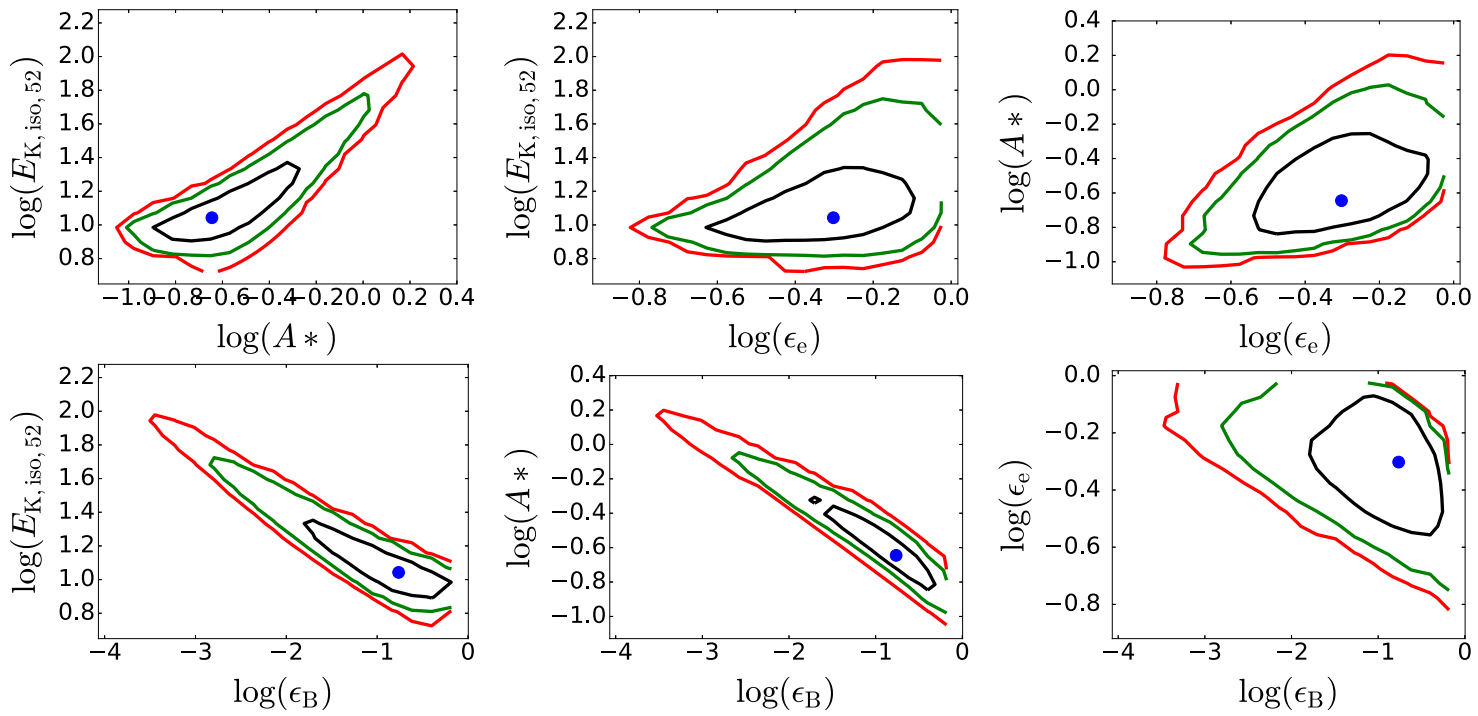

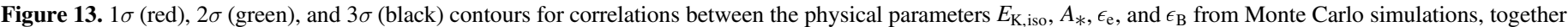
with the best-fit model (blue dot). We have restricted $\epsilon_{\mathrm{e}}+\epsilon_{\mathrm{B}}<1$, and do not include the radio data before 2.5 days in the analysis.

note that increased opacity at radio wavelengths is expected when non-shock accelerated electrons are present (Eichler \& Waxman 2005; Warren et al. 2017), and the increased self-absorption from these "thermal electrons" is indeed expected to decline with time
(Ressler \& Laskar 2017). A detailed analysis of this effect requires a treatment of the observed radiation spectrum, including thermal electrons in jetted GRB afterglows, and is beyond the scope of this work. 


\section{ORCID iDs}

Tanmoy Laskar (iD https://orcid.org/0000-0003-1792-2338

Edo Berger (iD https://orcid.org/0000-0002-9392-9681

Raffaella Margutti (iD https://orcid.org/0000-0003-4768-7586

Wen-fai Fong (iD https://orcid.org/0000-0002-7374-935X

B. Ashley Zauderer (i) https://orcid.org/0000-00031152-518X

\section{References}

Ade, P. A. R., Aghanim, N., Arnaud, M., et al. 2016, A\&A, 594, A13 Alexander, K. D., Laskar, T., Berger, E., et al. 2017, arXiv:1705.08455 Barthelmy, S. D., Barbier, L. M., Cummings, J. R., et al. 2005, SSRv, 120, 143 Berger, E., Kulkarni, S. R., \& Chevalier, R. A. 2002, ApJL, 577, L5 Bromm, V., Yoshida, N., \& Hernquist, L. 2003, ApJL, 596, L135 Burrows, D. N., Hill, J. E., Nousek, J. A., et al. 2005, SSRv, 120, 165 Cenko, S. B., Frail, D. A., Harrison, F. A., et al. 2010, ApJ, 711, 641 Cenko, S. B., Frail, D. A., Harrison, F. A., et al. 2011, ApJ, 732, 29 Chakraborti, S., Soderberg, A., Chomiuk, L., et al. 2015, ApJ, 805, 187 Chandra, P., Cenko, S. B., Frail, D. A., et al. 2008, ApJ, 683, 924 Chandra, P., \& Frail, D. A. 2012, ApJ, 746, 156

Chevalier, R. A. 1998, ApJ, 499, 810

Chevalier, R. A., \& Fransson, C. 2006, ApJ, 651, 381

Chornock, R., Berger, E., Fox, D. B., et al. 2013, ApJ, 774, 26

Chornock, R., Berger, E., Fox, D. B., et al. 2014, arXiv:1405.7400

Cucchiara, A., Levan, A. J., Fox, D. B., et al. 2011, ApJ, 736, 7

D’Avanzo, P., D'Elia, V., Piranomonte, S., et al. 2014a, GCN, 15964, 1

D’Avanzo, P., Melandri, A., Malesani, D., et al. 2014b, GCN, 15953, 1

de Ugarte Postigo, A., Lundgren, A., Martín, S., et al. 2012, A\&A, 538, A44

Duffell, P. C., \& Laskar, A. 2017, arXiv:1710.07253

Eichler, D., \& Waxman, E. 2005, ApJ, 627, 861

Evans, P. A., Beardmore, A. P., Page, K. L., et al. 2007, A\&A, 469, 379

Evans, P. A., Beardmore, A. P., Page, K. L., et al. 2009, MNRAS, 397, 1177

Fong, W., Berger, E., Metzger, B. D., et al. 2014, ApJ, 780, 118

Frail, D. A., Waxman, E., \& Kulkarni, S. R. 2000, ApJ, 537, 191

Fryer, C. L., Woosley, S. E., \& Heger, A. 2001, ApJ, 550, 372

Gehrels, N., Chincarini, G., Giommi, P., et al. 2004, ApJ, 611, 1005

Ghisellini, G., Nardini, M., Ghirlanda, G., \& Celotti, A. 2009, MNRAS, 393, 253

Granot, J., \& Piran, T. 2012, MNRAS, 421, 570

Granot, J., \& Sari, R. 2002, ApJ, 568, 820

Greiner, J., Krühler, T., Fynbo, J. P. U., et al. 2009, ApJ, 693, 1610

Haislip, J. B., Nysewander, M. C., Reichart, D. E., et al. 2006, Natur, 440, 181

Heger, A., Fryer, C. L., Woosley, S. E., Langer, N., \& Hartmann, D. H. 2003, ApJ, 591, 288

Holland, S. T., \& Racusin, J. L. 2014, GCN, 15973, 1

Hook, I. M., Jørgensen, I., Allington-Smith, J. R., et al. 2004, PASP, 116, 425

Inoue, S., Omukai, K., \& Ciardi, B. 2007, MNRAS, 380, 1715

Kawai, N., Kosugi, G., Aoki, K., et al. 2006, Natur, 440, 184

Klotz, A., Turpin, D., MacPherson, D., et al. 2014, GCN, 15952, 1

Kobayashi, S., Mészáros, P., \& Zhang, B. 2004, ApJL, 601, L13

Krimm, H. A., Barthelmy, S. D., Baumgartner, W. H., et al. 2014, GCN, 15962,1

Laskar, T., Alexander, K. D., Berger, E., et al. 2016, ApJ, 833, 88

Laskar, T., Berger, E., Margutti, R., et al. 2015, ApJ, 814, 1

Laskar, T., Berger, E., Tanvir, N., et al. 2014, ApJ, 781, 1

Laskar, T., Berger, E., Zauderer, B. A., et al. 2013, ApJ, 776, 119

Lemoine, M. 2015, MNRAS, 453, 3772
Li, L., Liang, E.-W., Tang, Q.-W., et al. 2012, ApJ, 758, 27

Liang, E.-W., Racusin, J. L., Zhang, B., Zhang, B.-B., \& Burrows, D. N. 2008, ApJ, 675, 528

Liang, E.-W., Zhang, B.-B., \& Zhang, B. 2007, ApJ, 670, 565

Lien, A., Sakamoto, T., Barthelmy, S. D., et al. 2016, ApJ, 829, 7

Littlejohns, O., Butler, N., Watson, A. M., et al. 2014, GCN, 15978, 1

Margutti, R., Genet, F., Granot, J., et al. 2010, MNRAS, 402, 46

Margutti, R., Zaninoni, E., Bernardini, M. G., et al. 2013, MNRAS, 428, 729

McMullin, J. P., Waters, B., Schiebel, D., Young, W., \& Golap, K. 2007, in ASP Conf. Ser 376, Astronomical Data Analysis Software and Systems XVI, ed. R. A. Shaw, F. Hill, \& D. J. Bell (San Francisco, CA: ASP), 127 Mészáros, P. 2006, RPPh, 69, 2259

Nardini, M., Ghisellini, G., Ghirlanda, G., \& Celotti, A. 2010, MNRAS, 403, 1131

Nava, L., Sironi, L., Ghisellini, G., Celotti, A., \& Ghirlanda, G. 2013, MNRAS, 433, 2107

Nousek, J. A., Kouveliotou, C., Grupe, D., et al. 2006, ApJ, 642, 389

Panaitescu, A., \& Kumar, P. 2002, ApJ, 571, 779

Pei, Y. C. 1992, ApJ, 395, 130

Perley, D. A., Cenko, S. B., Corsi, A., et al. 2014, ApJ, 781, 37

Piran, T. 2005, RvMP, 76, 1143

Prochaska, J. X., Chen, H.-W., Dessauges-Zavadsky, M., \& Bloom, J. S. 2007, ApJ, 666, 267

Racusin, J. L., Baumgartner, W. H., Gehrels, N., et al. 2014, GCN, 15944, 1

Ressler, S. M., \& Laskar, T. 2017, ApJ, 845, 150

Rhoads, J. E. 1999, ApJ, 525, 737

Roming, P. W. A., Kennedy, T. E., Mason, K. O., et al. 2005, SSRv, 120, 95

Salvaterra, R., Della Valle, M., Campana, S., et al. 2009, Natur, 461, 1258

Sari, R. 1997, ApJL, 489, L37

Sari, R., \& Esin, A. A. 2001, ApJ, 548, 787

Sari, R., Piran, T., \& Narayan, R. 1998, ApJL, 497, L17

Sault, R. J., Teuben, P. J., \& Wright, M. C. H. 1995, in ASP Conf. Ser. 77, Astronomical Data Analysis Software and Systems IV, ed. R. A. Shaw, H. E. Payne, \& J. J. E. Hayes (San Francisco, CA: ASP), 433

Schulze, S., Klose, S., Björnsson, G., et al. 2011, A\&A, 526, A23

Shao, L., \& Dai, Z. G. 2007, ApJ, 660, 1319

Suwa, Y., \& Ioka, K. 2011, ApJ, 726, 107

Tagliaferri, G., Antonelli, L. A., Chincarini, G., et al. 2005, A\&A, 443, L1

Tanvir, N. R., Fox, D. B., Levan, A. J., et al. 2009, Natur, 461, 1254

Tanvir, N. R., Levan, A. J., Wiersema, K., \& Cucchiara, A. 2014, GCN, 15961,1

Toma, K., Sakamoto, T., \& Mészáros, P. 2011, ApJ, 731, 127

Totani, T., Kawai, N., Kosugi, G., et al. 2006, PASJ, 58, 485

Uhm, Z. L., Zhang, B., Hascoët, R., et al. 2012, ApJ, 761, 147

Wang, F. Y., Bromm, V., Greif, T. H., et al. 2012, ApJ, 760, 27

Warren, D. C., Ellison, D. C., Barkov, M. V., \& Nagataki, S. 2017, ApJ, 835,248

Waxman, E., Kulkarni, S. R., \& Frail, D. A. 1998, ApJ, 497, 288

Willingale, R., Starling, R. L. C., Beardmore, A. P., Tanvir, N. R., \& O'Brien, P. T. 2013, MNRAS, 431, 394

Woosley, S. E., \& Bloom, J. S. 2006, ARA\&A, 44, 507

Xu, D., Bai, C.-H., Zhang, X., Esamdin, A., \& Ma, L. 2014a, GCN, 15956, 1

Xu, D., Bai, C.-H., Zhang, X., Esamdin, A., \& Ma, L. 2014b, GCN, 15947, 1

Yoshida, M., Itoh, R., Moritani, Y., et al. 2014, GCN, 15954, 1

Yost, S. A., Harrison, F. A., Sari, R., \& Frail, D. A. 2003, ApJ, 597, 459

Zaninoni, E., Bernardini, M. G., Margutti, R., Oates, S., \& Chincarini, G. 2013, A\&A, 557, A12

Zhang, W., \& MacFadyen, A. 2009, ApJ, 698, 1261 